\title{
LA CONTRACEPCIÓN EN EL TERCER MUNDO
}

\author{
LÉON TABAH * \\ Instituto Nacional de Estudios Demográficos, \\ Paris
}

La FECundrDad EFEctiva de una población es un hecho más bien social que fisiológico o aun sexual. El objetivo de la acción que se lleva a cabo en el Tercer Mundo es precisamente el de liberar el fenómeno de su raigambre social, de la inercia del cuadro sociocultural, por medio de la difusión de procedimientos nuevos como el dispositivo intrauterino, que estén de acuerdo con el conjunto limitado de motivaciones que prevalece en las poblaciones de gran pobreza, casi totalmente ausentes de aspiraciones y sin esperanza alguna de cambio.

No se debe perder de vista que la limitación de los nacimientos en las sociedades industriales ha tenido desde su origen un carácter burgués fundado en la idea, frecuentemente implícita y no percibida por los actores mismos, de que una descendencia numerosa podría entrar en conflicto con el deseo de desempeñar un cierto papel y lograr determinada posición (status) en una sociedad en constante transformación. Todas estas motivaciones han sido suficientemente fuertes para llevar a los pueblos de los países desarrollados a un dominio más o menos fuerte de la sexualidad por medio de ciertos procedimientos, desde luego constrictivos pero a la vez simples, que han tenido el mérito de no requerir ser "descubiertos" y de no exigir difusión, ya que vienen naturalmente a la mente de aquellos que sien. ten la necesidad. Por otra parte, el "terreno" estaba preparado en gran parte por la ética puritana altamente racionalista y maltusiana de los anglosajones, los escandinavos y los protestantes europeos y por la actitud individualista que caracterizaba la cultura francesa en vísperas de la Revolución, en la parte de Europa donde la influencia francesa era preponderante.

Era poco probable que a los pueblos menos desarrollados les satisficieran los mismos procedimientos; por ello las investigaciones de los laboratorios de Europa occidental y los Estados Unidos se han orientado por la idea de que se deben encontrar procedimientos susceptibles de ser aceptados por poblaciones en las que las motivaciones se encuentren a niveles bastante débiles. De los dos procedimientos encontrados, uno de ellos es un esterilizante mecánico (el dispositivo intrauterino) y el otro un esterilizante químico (la píldora). EI pri-

* Este artículo aparecerá en francés en la revista Population, del Institut National d'Études Démographiques (INED), París. Traducción del francés por Enrique Brito, revisada por la Redacción. 
mero tiene, más que el segundo, la ventaja de poder ser considerado "acultural" en el sentido de que puede ser implantado cualquiera que sea el sistema de valores. De hecho, se ha difundido en tipos culturales tan variados como los de la India, el Medio Oriente y la América Latina.

Las cuestiones que se presentan son numerosas, pues el problema se plantea en diversas dimensiones a la vez: fisiológica, psicosociológica, moral y política. Existe toda una álgebra de la fecundidad de las poblaciones humanas que constituye una verdadera madeja de variables difícil de desembrollar para determinar la coherencia y la lógica de base, tanto sobre el plano cuantitativo como sobre el cualitativo.

\section{LA FECUNDIDAD NATURAL}

Recordemos en primer término cuál sería la fecundidad de una población que no recurriera a la contracepción o, lo que viene a ser lo mismo, que la practicara con eficacia nula. Los resultados de numerosas investigaciones sobre el tema, debidas sobre todo a L. Henry, R. Potter, C. Tietze y M. Sheps, han sido relacionados entre sí por medio de un modelo de simulación presentado por A. Jacquard en la revista Population [1]. ${ }^{1}$ Este estudio demuestra que en las condiciones de nupcialidad y de mortalidad de las poblaciones europeas de hoy, la mitad de las mujeres tendría cuando menos diez nacimientos, y que la media sería de 8.6 ; la tasa de crecimiento anual de tal población sería de $4.4 \%$.

De hecho, las tasas de crecimiento en el Tercer Mundo son inferiores a esta cifra, siendo la media del orden de $2.4 \%$ y la tasa máxima de $3.7 \%$. La diferencia se explica por una práctica contraceptiva en ciertas capas sociales, por el aborto que no se tuvo en cuenta en el modelo, y, sobre todo, por las condiciones sanitarias menos adecuadas que las de los países europeos. En cambio, la tasa de nupcialidad actúa en forma favorable a una fecundidad más elevada y, en consecuencia, a tasas de crecimiento también más elevadas.

Como conclusión de todo esto se puede afirmar que si el descenso de la mortalidad continúa sin que la nupcialidad y la fecundidad se modifiquen, los países del Tercer Mundo están llamados a experimentar tasas de crecimiento demográfico todavía superiores a las actuales.

¿Qué puede esperarse, en el Tercer Mundo, de la difusión de los nuevos procedimientos de contracepción y en particular del dispositivo intrauterino? Antes de examinar este punto conviene recordar algunas informaciones esenciales concernientes al procedimiento.

\section{EL DISPOSITIVO INTRAUTERINO}

La práctica contraceptiva consistente en introducir un cuerpo extraño en el útero es bien antigua. A. L. Southam [2], observa que la literatura médica sobre el tema es abundante a lo largo del siglo xix. Pero esta práctica no fue presentada en forma sistemática sino hasta

1 Véanse las referencias bibliográficas al final. 
1928 en Berlín, ante un grupo de médicos, por el doctor E. Gräfenberg [3], y produjo verdadera conmoción entre los ginecólogos tanto de Estados Unidos como de Alemania.2

La técnica propuesta por Gräfenberg consistía en reemplazar los contraceptivos uterinos de crin, llamado crin de Florencia, que entonces se conocían, a menudo en forma de estrella, por anillos de oro o de plata visibles bajo rayos X. Es interesante hacer notar que Gräfenberg pensaba desde entonces que ese método podía convenir a las mujeres pertenecientes a los medios sociales menos favorecidos. Emigrado a los Estados Unidos en 1940, tuvo que renunciar, ante la oposición de los médicos norteamericanos, a difundir su método, y desde entonces casi no se ha vuelto a hablar de éste. En 1955, un informe sobre el tema presentado en la $\mathrm{V}$ Conferencia Internacional sobre Planificación Familiar, en Tokio, apenas si despertó atención.

Dos publicaciones provocaron simultáneamente la renovación del interés por los contraceptivos intrauterinos en 1959. Primero, un artículo en el que W. Oppenheimer [6] presentó los resultados de experimentos hechos a lo largo de veintiocho años en Israel. En un principio se usó el anillo de plata, pero fue remplazado en el curso de las investigaciones por el antiguo anillo de crin. Un grupo de mujeres se prestó a esta experiencia a lo largo de veinte años. Las investigaciones de Oppenheimer se basaron en 329 mujeres que representaban un total de 793 años de exposición. Sus conclusiones fueron favorables al método, tanto desde el punto de vista de la salud como de la eficacia; se registraron sólo 20 embarazos en total.

La otra publicación que de modo independiente despertó de nuevo el interés por los contraceptivos intrauterinos fue un artículo en el que A. Ishihama [7] dio cuenta de 18594 observaciones en 149 hospitales japoneses. Se empleó desde 1934 un anillo metálico (anillo de Ota), ligeramente diferente al de Gräfenberg; se usaron también contraceptivos intrauterinos de polietileno. Al parecer, en esta última publicación se menciona por primera vez el dispositivo intrauterino de nylon que ha tenido ya tanto éxito en el Tercer Mundo.

A partir de entonces, el interés por este método se ha extendido sin cesar, y se le consagró íntegramente en un congreso internacional en 1962, con participación de unos cincuenta especialistas de Estados Unidos, Inglaterra, Puerto Rico, México, Chile, Egipto, Israel, Japón, Formosa, India y Paquistán [8].

Cabe mencionar, entre los trabajos presentados, los consagrados a nuevas formas del dispositivo intrauterino: espirales de Margulies, doble S de Lippes, "nudos" de Birnberg, todos de material plástico y tamaño variable, salvo el anillo de acero inoxidable de Hall-Stone. El material plástico permite, a diferencia del anillo metálico de Gräfenberg, una permanencia casi indefinida del dispositivo en el útero, cuando no es expulsado, y su flexibilidad presenta ventajas evidentes tanto para la colocación como para la extracción. Las conclusiones

2 Se encontrará una bibliografía sobre los contraceptivos intrauterinos, en el período 1920-1965, que se podría calificar de completa, en una obra de A. VellayDalsace [4] y un estudio histórico de este método contraceptivo en un artículo de C. Tietze [5]. 
de este congreso fueron tan alentadoras que se aprobó una resolución en la sesión de clausura a favor de nuevas investigaciones clínicas en forma lo más coordinada posible.

En octubre de 1964 se llevó a cabo en Nueva York un segundo congreso internacional sobre el mismo tema que aportó una nueva y abundante cosecha de observaciones. Los trabajos presentados se dividieron en dos temas esenciales: 1) estudios clínicos e investigaciones sobre el terreno, y 2) estudios biomédicos [9].

Han transcurrido casi cuarenta años desde el primer artículo de Gräfenberg y todavía no han sido enteramente dilucidados los mecanismos de operación del dispositivo intrauterino. No es ésta ciertamente la primera vez que se recurre a acciones cuyo mecanismo de fondo se ignora, pero el problema requiere una importancia particular en razón de su incidencia filosófica, moral y religiosa. En particular, se plantea en el aspecto moral el problema de determinar en qué momento tiene lugar esta acción: $¿$ antes o después del comienzo del embarazo, esto es, de la vida? Pero, si como las investigaciones recientes parecen permitirlo, se puede saber en qué momento del proceso reproductivo interviene la acción del dispositivo intrauterino, queda en pie otra cuestión de importancia: ¿cuándo tiene lugar el comienzo del embarazo? ¿En el momento en que el óvulo es fecundado o en el momento de la anidación?

Para algunos, como el mismo Gräfenberg, el acceso de la esperma a las trompas no es impedido por el dispositivo; pero, en cambio, la anidación se hace imposible, habiéndose comprobado casos de embarazo extrauterino. Para otros, como Zipper, que trabajó en este asunto desde 1959, se producen alteraciones bioquímicas que impiden la concepción; en tanto que para numerosos autores -Margulies y Doyle, Guttmacher, Tietze - la presencia de un cuerpo extraño en el útero provoca un peristaltismo de las trompas en el momento de la ovulación, de suerte que el óvulo, aunque esté fecundado, llega prematuramente al útero no preparado y no se puede implantar ahí. Investigaciones recientes hechas sobre monas por L. Mastroiani y C. Hongsanand [10] parecen, en efecto, indicar que el óvulo que permanece normalmente unos tres días en las trompas de Falopio, recorre muy rápidamente esta zona cuando se coloca un cuerpo extraño en el útero. Ésta es en la actualidad la tesis que parece reconocerse como la más autorizada.

Se estima que a fines de 1966 el número de inserciones del dispositivo intrauterino era de unos 3 millones en Asia (de ellos, 1250000 en la India), 100000 en el Cercano Oriente y África del Norte, y 155000 en América Latina. En cambio, no hay idea precisa del número de inserciones en Europa, Estados Unidos, la parte sur de África, Japón y China continental. En relación con el número de mujeres de 20 a 44 años, es en Corea del Sur donde la proporción de las portadoras es mayor $(17 \%)$, seguida de Formosa (13\%). En Paquistán la proporción era de $3 \%$ únicamente, y lo mismo en la India. Cabe hacer notar el rápido aumento de las inserciones: de 318000 en la India en 1965 se ha pasado a 1250000 en 1966 [11]. 


\section{LA CONTRACEPCIÓN POR LA VÍA ORAL (LA PÍLDORA)}

Se hará apenas breve referencia al empleo con fines contraceptivos de los progestativos sintéticos, porque hasta ahora está poco extendido en el Tercer Mundo. Desde 1937, Makepeace, Weinstein y Friedman demostraron por experimentos en animales que la progesterona inhibía la función ovárica. Se demostró igualmente que otros esteroides producían el mismo efecto. No fue sino hasta 1956 cuando Rock, García y Pincus demostraron en forma indiscutible la eficacia de estos productos en la regulación de la fecundidad humana, y los experimentos comenzaron ese mismo año en San Juan, Puerto Rico, durante dieciocho meses, con 265 mujeres casadas [12]. De allí en adelante, el éxito del método se ha multiplicado sin cesar, y se estima en muchas decenas de millones el número de mujeres que recurren a él. Existe sobre el tema abundante literatura.

\section{Evaluación deL MÉTodo DEL DispositTyo INTRAUTERINo}

Se tratará de hacer una evaluación del método del dispositivo intrauterino en el Tercer Mundo analizando las informaciones disponibles, desde el punto de vista de los fracasos, las expulsiones, las extracciones y los efectos secundarios; estas informaciones se compararán con las de Estados Unidos, único país industrializado que ha publicado observaciones sobre todos estos puntos.

Mayor eficacia que por los procedimientos tradicionales. Desde luego, la eficacia de los nuevos procedimientos parece ser bien superior a la de los tradicionales. La eficacia de un contraceptivo se mide normalmente por la proporción de fracasos, es decir, la relación entre el número de embarazos no deseados, en un lapso determinado, y el número de años de exposición durante el mismo período.

En lo que concierne al método de cálculo, los autores recurren en general al propuesto por R. G. Potter [13], que se puede resumir de la siguiente manera: las proporciones de fracaso $q_{x}$ relativas a cada mes $x$ se calculan en razón de la relación entre los fracasos observados durante el mes $x$ y el número de mujeres sometidas al riesgo de fecundidad al comienzo de ese mes $x$. Se calculan en seguida los complementos a 1 , es decir, $p_{x}=1-q_{x}$, y los productos $P_{x}=p_{1} p_{2} \ldots p_{x}$. Esta cantidad $P_{x}$ indica la proporción teórica de mujeres que no habrían tenido fracasos después de $x$ meses si hubieran estado sometidas durante todo el período al riesgo de fecundidad determinado por la ley $q_{x}$. El complemento a 1 , o sea $Q_{x}=1-P_{x}$, indica la proporción teórica, con relación al número inicial de mujeres, de las que habrían sufrido fracasos después de $x$ meses. Esta proporción puede ser expresada en meses-mujer o en años-mujer.

El procedimiento, como se ve, es muy semejante al de la tabla de mortalidad, sólo que las bajas por mortalidad quedan remplazadas por las bajas debidas a los fracasos de la contracepción. Hay, sin embargo, una diferencia importante entre los dos tipos de razonamiento en el sentido de que no se desaparece de la tabla de sobre- 
vivientes sino por muerte, en tanto que se puede egresar del grupo inicial de mujeres consideradas en el cálculo de la eficacia de un método contraceptivo no sólo por razón de fracaso, sino también, para un método como el del dispositivo intrauterino, por otras causas que distan de ser despreciables: deseo de maternidad, abandono o cambio del método contraceptivo, suspensión por razones médicas, expulsión, fallecimiento, etc.; la interpretación de las proporciones de fracaso es por lo tanto más delicada que la función que le corresponde en las tablas de sobrevivientes.

Para remediar esta dificultad, C. Tietze [14] calcula dos tipos de tasas, que denomina "netas" y "brutas". Las primeras toman en cuenta otros riesgos y son aditivas. Por ejemplo, una tasa neta de fracaso es afectada por las expulsiones o extracciones del dispositivo; la suma de las tasas netas de fracaso, de expulsión y de extracción da la tasa de bajas de observación con respecto a una de estas tres causas. Por el contrario, la tasa bruta de fracaso es la tasa teórica que se observaría en ausencia de todo otro riesgo de baja en la observación.

Es interesante hacer notar que casi todos los cálculos que se mencionan en este artículo, ya se trate de proporciones de fracaso o de expulsión o extracción, se basan al parecer en los mismos métodos, lo que permite así la comparación de los resultados.

Es claro que la eficacia de un contraceptivo depende en buena parte de la habilidad de la persona, de la fuerza de sus motivaciones y de la aceptabilidad del método. Pero en el caso de nuevos contraceptivos, sobre todo el dispositivo intrauterino -y este punto es de importancia decisiva-, depende también de la calidad del sistema médico-social, en la medida en que éste permita visitas suficientemente repetidas de las mujeres. La eficacia puede, por lo tanto, variar bastante de un medio social a otro, y entre los países industrializados y los subdesarrollados.

Veamos primero cuáles son las proporciones de fracaso de los llamados procedimientos tradicionales en las poblaciones cuyas informaciones permiten hacer el cálculo (Estados Unidos). En la encuesta de Indianapolis, que se llevó a cabo entre parejas de raza blanca, con 12 a 15 años de vida matrimonial, lo que implica desde luego que las mujeres eran de edad relativamente avanzada, R. G. Potter [15] obtuvo una tasa de fracasos para el conjunto de los métodos (preservativo, diafragma y jaleas, coito interrumpido, ritmo, ducha postcoital y combinación de estos métodos), de 21.5 por cien años-mujer. En la encuesta, llamada de Princeton o también "American Family Growth", que se efectuó entre 1165 parejas de raza blanca residentes en siete sectores muy urbanizados, sometidas a un interrogatorio doble - el primero seis meses después del nacimiento de un segundo hijo en septiembre de 1956 y el otro al comienzo de 1960, lo que permitió un análisis parcial longitudinal-, el mismo autor obtuvo, respecto al conjunto de métodos contraceptivos, una proporción de 19.9 por cien añosmujer; es decir, bastante semejante a la encuesta de Indianapolis. Hay, desde luego, fuertes variaciones en las tasas de fracaso, según los métodos, siendo la más baja de $13.8 \%$ para el preservativo, y la más elevada de $40.8 \%$ para la ducha post-coital. 
En lo que se refiere a los progestativos sintéticos, las proporciones de fracaso encontradas hasta ahora, aplicando el mismo método de cálculo [16], no parecen exceder del $1.7 \%$. (Véase el cuadro 1.)

Cuadro 1

NÚMERO DE FRACASOS DE LOS PROGESTATIVOS POR 100 AÑOS-MUJER

\begin{tabular}{|c|c|c|c|c|c|}
\hline Autor & $\begin{array}{l}\text { Año de } \\
\text { publi- } \\
\text { cacion }\end{array}$ & $\begin{array}{l}\text { Lugar de la } \\
\text { investiga } \\
\text { cion }\end{array}$ & $\begin{array}{l}\text { Núrero de } \\
\text { parejas }\end{array}$ & $\begin{array}{c}\text { Mumero de } \\
\text { meses de } \\
\text { exposi- } \\
\text { cion }\end{array}$ & $\begin{array}{l}\text { Prcpor- } \\
\text { cior de } \\
\text { fracasos } \\
(\%)\end{array}$ \\
\hline $\begin{array}{l}\text { Coldzieher y otros } \\
\text { Watts y Diddle } \\
\text { Kice Wray y otros } \\
\text { Rears } \\
\text { Tyler y otros } \\
\text { Cook y otros } \\
\text { Pincus }\end{array}$ & $\begin{array}{l}1962 \\
1962 \\
1962 \\
1962 \\
1962 \\
1961 \\
1961\end{array}$ & $\begin{array}{l}\text { San Antonio (E.U.) } \\
\text { Knoxville (E.U.) } \\
\text { México, D.F. } \\
\text { Gran Bretaña } \\
\text { Los Angeles (E.U.) } \\
\text { Puerto Rico } \\
\text { Puerto Rico y Haiti }\end{array}$ & $\begin{array}{r}210 \\
214 \\
364 \\
391 \\
570 \\
550 \\
1500\end{array}$ & $\begin{array}{rr}6 & 139 \\
3 & 050 \\
6 & 062 \\
3 & 128 \\
7 & 194 \\
6 & 734 \\
25 & 421\end{array}$ & $\begin{array}{l}0 \\
0.4 \\
0.6 \\
0.8 \\
1.1 \\
1.4 \\
1.7\end{array}$ \\
\hline
\end{tabular}

Fuente: Ver referencia [16].

Con relación a los contraceptivos intrauterinos, se indican en el cuadro 2 las proporciones de fracaso de las diferentes formas de dispositivos intrauterinos, en distintos lugares.

Se advierte que las proporciones de fracaso de los nuevos procedimientos de contracepción son, en Estados Unidos, muy inferiores a las de los procedimientos tradicionales. La tasa parece ser un poco inferior en el caso de los esterilizantes químicos ( 1.7 por cien añosmujer, cuando más), que en el del dispositivo intrauterino (2.4 a 5.3 por cien años-mujer en Estados Unidos) si se excluyen los dispositivos en forma de nudo y en forma de espiral, que no parece que deban utilizarse en el futuro a menos que se les hagan modificaciones, como se verá más adelante. ${ }^{3}$

Pero en la interpretación de estas cifras hay que tener presente que las observaciones relacionadas con el dispositivo intrauterino en los Estados Unidos corresponden a una población en que la importancia y la calidad del aparato médico-social permite exámenes frecuentes de las mujeres. Las expulsiones, que son a menudo la causa de los fracasos cuando pasan desapercibidas de la portadora, se conocen así bien pronto. ${ }^{4}$

Las tasas de fracaso calculadas hasta ahora en los países subdesarrollados no parecen diferir mucho de las obtenidas en los Estados Unidos, pero se trata de experimentos limitados a zonas relativamente

3 Es probable que la proporción de fracasos disminuya hasta un 2.5 por cien años-mujer si, como parece, no se conservan más que los dispositivos doble $\mathrm{S}$, tallas C. y D. Las tasas de fracaso de los contraceptivos intrauterinos se aproximarán a las de la píldora.

4 En caso de fracaso, el dispositivo no es necesariamente extraído del útero. Puede permanecer ahí hasta el parto o, eventualmente, el falso parto, sin que hasta ahora se hayan verificado efectos nocivos sobre la madre durante el embarazo o el parto, o sobre el feto, aunque no se ha hecho ningún estudio detallado sobre los niños nacidos en estas condiciones. 
Cuadro 2

VARIOS PAÍses: PROPORCIONES DE FRACASO, POR 100 INSERCIONES, DE LOS CONTRACEPTIVOS INTRAUTERINOS, SEGÚN FORMA Y TALLA

\begin{tabular}{|c|c|c|c|c|c|c|c|c|}
\hline & \multicolumn{2}{|c|}{$\begin{array}{l}\text { Estados Unidos } \\
(1963-1966)\end{array}$} & \multirow[t]{2}{*}{$\begin{array}{c}\text { Formosa } \\
\text { (Taichung) } \\
(1963- \\
1965) \\
\end{array}$} & $\begin{array}{l}\text { Puerto } \\
\text { Rico } \\
(1962- \\
1964) \\
\end{array}$ & $\begin{array}{c}\text { Corea } \\
\text { de1 Sur } \\
(1962- \\
1964)\end{array}$ & $\begin{array}{c}\text { Chile } \\
\text { (Santiago) } \\
(1959- \\
1963)\end{array}$ & \multirow[t]{2}{*}{$\begin{array}{c}\text { Hong } \\
\text { Kong } \\
(1963- \\
1964) \\
\end{array}$} & \multirow[t]{2}{*}{$\begin{array}{r}\text { Jamaica } \\
(1964- \\
1965) \\
\end{array}$} \\
\hline & \multicolumn{2}{|c|}{$\begin{array}{c}\text { Al fin de: } \\
\text { Primer Segundo } \\
\text { año año } \\
\end{array}$} & & \multicolumn{3}{|c|}{ Al fin del primer año } & & \\
\hline $\begin{array}{l}\text { Número de in- } \\
\text { serciones } \\
\text { número de meses }\end{array}$ & \multicolumn{2}{|c|}{29.753} & 3.719 & 1.396 & 3.429 & 3.669 & 3.238 & 1.316 \\
\hline $\begin{array}{l}\text { de exposición } \\
\text { Doble S (10op) }\end{array}$ & \multicolumn{2}{|c|}{381.627} & $?$ & 12.970 & 12.262 & 49.644 & 8.237 & 8.062 \\
\hline $\begin{array}{l}\text { Talla.A } \\
(25 \mathrm{~mm}) \\
\text { Talla B }\end{array}$ & 5.3 & 9.3 & 6.9 & 6.8 & 6.2 & & & \\
\hline $\begin{array}{l}(31 \mathrm{~mm}) \\
\text { Talla C }\end{array}$ & 2.5 & 4.8 & 2.4 & & ' & & & \\
\hline $\begin{array}{l}(30 \mathrm{~mm}) \\
\mathrm{Ta} 1 \mathrm{Da}\end{array}$ & 2.4 & 4.0 & 1.0 & & & & & 3.6 \\
\hline$(27.5)$ & 2.6 & $4 . I$ & 2.4 & 1.5 & 2.3 & & 2.5 & \\
\hline$\frac{\text { Espiral }}{\text { Pequeña }}$ & 3.7 & 4.5 & & $6.4^{\circ}$ & & & & \\
\hline $\begin{array}{c}\text { Grande } \\
\text { Mudo (bow) }\end{array}$ & 1.4 & 2.2 & $3 . ?$ & 0.4 & & & & \\
\hline $\begin{array}{l}\text { Pequeño } \\
\text { Grande }\end{array}$ & $\begin{array}{r}10.8 \\
4.6\end{array}$ & $\begin{array}{r}16.1 \\
7.1\end{array}$ & & & . & & & \\
\hline $\begin{array}{l}\text { Anillo de ace- } \\
\text { ro }\end{array}$ & & 9.0 & & & & & & \\
\hline $\begin{array}{l}\text { Anilizo de } \\
\text { nylon }\end{array}$ & 6.6 & & & & & & & \\
\hline
\end{tabular}

Fuentes:

Estados Unidos: referencia [19].

Formosa: [17].

Puerto Rico: A. P. Satterthwaite, D. Arandes, M. E. Negron, "Experience with Intra-Uterine Devices in Puerto Rico", en [9], pp. 76-83.

Corea del Sur: H. Su Shin, "The Intra-Uterine Devices Program in Korea", en [9], pp. 45-51.

Chile: J. Zipper, M. L. García, L. L. Pastene, "Intra-Uterine Contraception with the Use of a Flexible Nylon Ring: Experience in Santiago de Chili", en [9], pp. 88-93.

Hong Kong: [35].

Jamaica: L. L. Williams, "Post-partum Insertion of a Standard Lippes Loop", en B. Berelson y otros [24].

bien supervisadas y equipadas en materia de salubridad pública. En particular, se dispone todavía de muy pocos informes sobre la India y Paquistán, en donde desde hace un año se ha hecho gran difusión del dispositivo intrauterino.

Llama la atención la gran diversidad de resultados, debida en gran parte, según parece, al modelo del dispositivo intrauterino, lo que no debe extrañar puesto que se encuentra todavía en fase experimental. Los datos recogidos en el curso de los últimos dos años, y que han sido objeto de publicaciones que han llegado a conocimiento del autor, indican tasas de fracaso que varían de 1.0 a 6.9 por cien años-mujer 
en Formosa, 2.3 a 6.2 en Corea del Sur, 1.5 a 6.8 en Puerto Rico, 4.5 en Santiago de Chile y 3.6 en Jamaica.

Cualquiera que sea la variabilidad de los resultados, las tasas de fracaso pasan muy raramente de $7 \%$, por el momento, en tanto que las observadas respecto a los procedimientos tradicionales en Europa son, a menudo, como se ha visto, dos o tres veces superiores. En Formosa [17] se han encontrado en dos grupos de mujeres que adoptaron en un caso los procedimientos tradicionales (sobre todo el preservativo, el método Ogino o espermicidas químicos), y en el otro el dispositivo intrauterino, durante un período de seis meses, una tasa de fracaso de 19.1 por cien años-mujer en el primer grupo, y de 3.4 en el segundo.

Parece lógico suponer que una difusión en gran escala del dispositivo intrauterino, en los países en que el aparato médico-social es todavía insuficiente para permitir exámenes frecuentes de las mujeres, conducirá a tasas de fracaso superiores a las encontradas hasta aquí. Pero todavía se pueden esperar mejorías en la técnica misma del contraceptivo uterino, por ejemplo, gracias a mejor adaptación de su forma a la morfología uterina. Esta adaptabilidad juega papel importante tanto desde el punto de vista de los fracasos como de las expulsiones, o de los efectos secundarios, como lo demuestran H. J. Davis y F. Israel [18].5

Sea como fuere, el problema de la eficacia no debería constituir un obstáculo insuperable para la difusión de los nuevos contraceptivos en el Tercer Mundo, aun en el caso de que ella disminuya al pasar de la fase experimental a la de difusión en gran escala. Más importantes parecen ser los inconvenientes relacionados con las expulsiones y con las extracciones motivadas por efectos secundarios, que se examinarán en el párrafo siguiente. En los países europeos y Estados Unidos, las parejas se han conformado con medios de limitación que son a la vez menos seguros y más frustrantes, de suerte que entre las poblaciones en que la fecundidad es muy elevada y en las cuales se supone, como todo lo permite suponer, un claro deseo de prevenir los nacimientos, puesto que los abortos son muy frecuentes, puede esperarse buena aceptación de los procedimientos cuyas tasas de fracaso se encuentren por término medio entre 5 y 10 por cien años-mujer; en especial porque las ventajas sobre los otros métodos -sobre todo la ausencia casi total de molestias en el plano sexual- son evidentes.

La frecuencia de las expulsiones y de las extracciones de contraceptivos intrauterinos no es despreciable. La tasa de expulsiones del dispositivo intrauterino varía considerablemente según la forma y el tamaño del dispositivo, pero en sentidos que son paralelos, por lo ge-

5 En los Estados Unidos se lleva a cabo en el National Committee on Maternal Health un experimento interesante: se colocaron dispositivos en forma de doble $\mathrm{S}$, de tres tallas diferentes, sin tener en cuenta la forma de la cavidad uterina ni las preferencias de los experimentadores, es decir, de manera aleatoria. Al darse a conocer el más reciente informe [19], la experiencia sólo tenía seis meses de duración, de suerte que nada podía deducirse aún.

Se consideró que se requeriría emplear dispositivos más pequeños en las mujeres asiáticas que en las europeas, pero la experiencia no ha confirmado esta hipótesis y se tiende en todos los casos hacia modelos más grandes que los utilizados en lin principio. 
neral, a la de los fracasos. La mitad de las expulsiones se producen en el curso de los primeros tres meses.

Las informaciones relativas a los Estados Unidos que se reproducen en el cuadro 3 son las del estudio de C. Tietze [19], que reúne las observaciones hechas con el apoyo del National Committee on Maternal Health por 38 investigadores que trabajaron en forma coordinada a fin de permitir la comparación de los resultados. Como se puede ver, las proporciones de expulsión varían en los Estados Unidos, según los modelos, de 1.6 a 19.8 por cien inserciones, en el curso del primer año y para el caso de primeras inserciones. Si se siguen empleando los modelos que parecen gozar del favor de los experimentadores, es decir, los de doble $\mathrm{S}$ de talla $\mathrm{C}(30 \mathrm{~mm})$ y $\mathrm{D}(31 \mathrm{~mm})$, las proporciones de expulsión son, respectivamente, de 12.1 y $9.1 \%$.

Los resultados correspondientes a estos dos últimos modelos parecen ser un poco menos satisfactorios en Formosa, donde se han encontrado proporciones respectivas de 16.0 y $13.4 \%$. En Puerto Rico, la tasa relativa al dispositivo intrauterino doble $S$ de talla $D$ es de $8.5 \%$ y por lo tanto muy cercana a la de Estados Unidos. Por el contrario, en Corea del Sur las proporciones de expulsión no pasan del $5 \%$. En Hong Kong, la proporción es del $10 \%$ para toda forma de dispositivo (doble $\mathrm{S}$ o espiral). En Jamaica, es de $8.2 \%$ para la doble $\mathrm{S}$ de talla $\mathrm{C}$. En la India, en donde el número reducido de observaciones no permite llegar a conclusiones valederas, la proporción es poco elevada $(8.0 \%$ para la espiral y $5.0 \%$ para la doble $\mathrm{S}$ ).

Las proporciones de expulsión son elevadas en Chile (17.2\%), donde el dispositivo intrauterino es un anillo de nylon, y por lo tanto diferente a los que se emplean en cualquier otra parte.

Las proporciones de nuevas expulsiones después de la reinserción son muy elevadas y pasan del $50 \%$ durante el primer año en los Estados Unidos [20].

Las proporciones de extracción (véase el cuadro 4) son bastante elevadas en Estados Unidos, donde varían de 12 a $14 \%$ por razones médicas (hemorragias, dolores, contracciones uterinas, inflamaciones pelvianas, etc.), en cuanto al dispositivo intrauterino en forma de doble $\mathrm{S}$, y de 3 a $6 \%$ cuando se trata de razones personales (deseo de maternidad, falta de confianza en el método, objeción del esposo, desaprobación del médico privado, etc.). Las tasas de extracción respecto a toda clase de causas alcanzan en los Estados Unidos de 15 a $18 \%$ para el dispositivo intrauterino en forma de doble S. Estas proporciones parecen ser más o menos del mismo orden en Puerto Rico. Son, por el contrario, más elevadas en Formosa, "en razón de la falta de experiencia de los médicos y de la falta de confianza en el método", según el último informe sobre este país. En Paquistán, en la experiencia piloto de Lulliani [21] sobre un número ciertamente bien reducido de inserciones (134), la proporción de expulsión o de extracción fue de $36.6 \%$ al final del primer año, y los experimentadores hacen notar que los datos recientes parecen indicar que tales proporciones se mantendrán a niveles elevados en el segundo y tercer año siguientes a la inserción. En cambio, las tasas de extracción son relativamente bajas en otras experiencias: Corea del Sur, Chile, Hong Kong, Jamaica e India. 
Cuadro 3

VARIOS países: Proporción de EXPUlsiones del dispositivo intrauterino DURANTE EL PRIMER AÑO

(Porcientos)

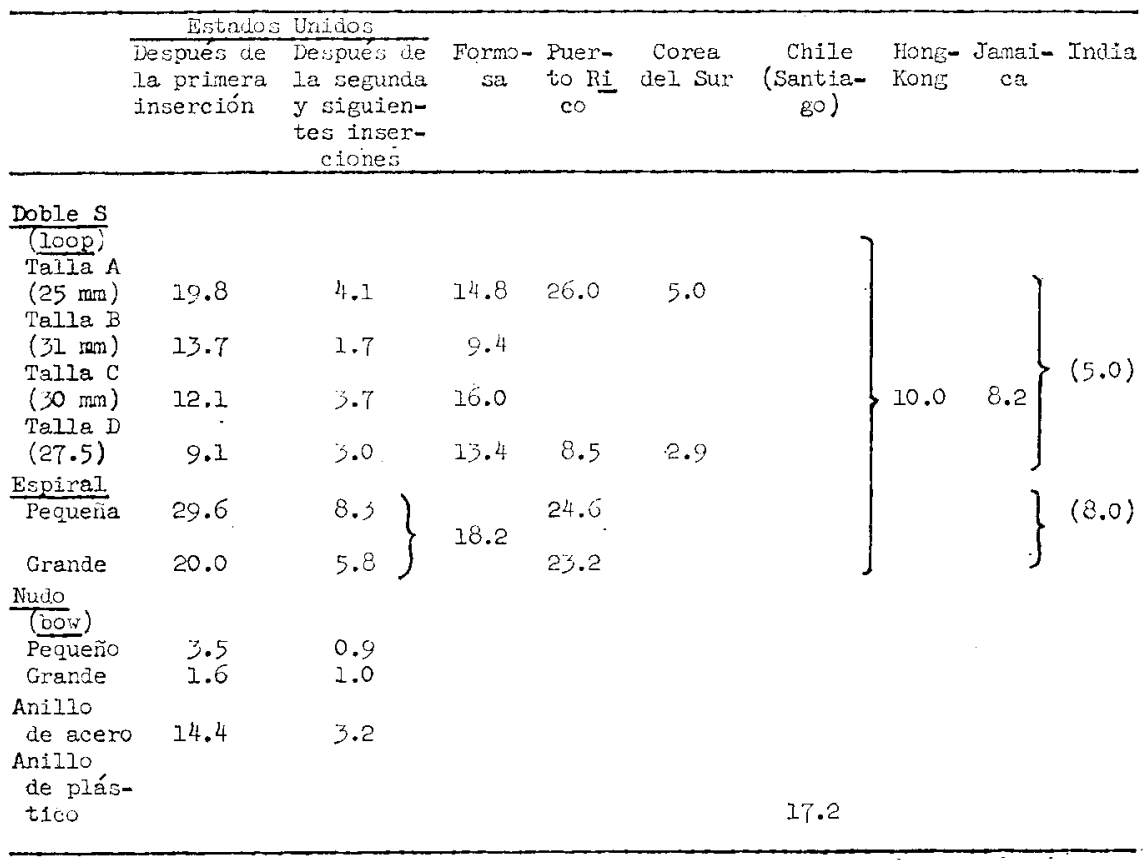

Fuentes: mismas citadas en el cuadro 2; India, misma que Hong Kong.

Nota: Las cifras entre paréntesis se refieren a muy escaso número de observaciones.

Las proporciones de expulsión, así como las de extracción, disminuyen sensiblemente con el número de partos y con la edad de la madre; y las de fracaso varían en sentido inverso, tanto en Estados Unidos como en Chile, siendo estos dos países los únicos que hasta ahora han proporcionado informaciones sobre el particular. Los especialistas están de acuerdo en desaconsejar el uso del dispositivo intrauterino a las mujeres que no han estado embarazadas todavía. El éxito del procedimiento es bastante débil en las mujeres jóvenes o con pocos hijos, y por el contrario, más preciso en las mujeres después del tercero o cuarto hijo.

Los experimentadores buscan un modelo que permita garantizar la mayor eficacia posible en todos los aspectos a la vez: seguridad, retención e inocuidad. Las investigaciones norteamericanas, que abarcan la mayor variedad de formas y de tallas de dispositivos intrauterinos, indican que la espiral grande presenta una tasa de fracaso de apenas $1.8 \%$ durante el primer año, pero una elevada proporción de expulsiones que llega al $20.0 \%$. Por el contrario, el pequeño nudo (smalt bow) presenta una proporción de expulsión de $3.5 \%$ en 


\section{Cuadro 4}

Varios países: Proporciones de eXtracción del dispositivo intrauterino durante el PRimer aÑo (Porcientos)

\begin{tabular}{|c|c|c|c|c|c|c|c|c|c|c|c|c|c|c|}
\hline & \multicolumn{3}{|c|}{ Estados Unidos } & \multirow[b]{2}{*}{$\begin{array}{c}\text { Formo } \\
\text { sa } \\
\text { Total } \\
\end{array}$} & \multicolumn{3}{|c|}{ Puerto Rico } & \multirow[b]{2}{*}{$\begin{array}{c}\text { Corea } \\
\text { del Sur } \\
\text { Total }\end{array}$} & \multicolumn{3}{|c|}{ Chile } & \multirow[b]{2}{*}{$\begin{array}{r}\text { Hong } \\
\text { Kong } \\
\text { Total } \\
\end{array}$} & \multirow[b]{2}{*}{$\begin{array}{c}\text { Jamai } \\
\text { ca } \\
\text { Total } \\
\end{array}$} & \multirow[b]{2}{*}{$\begin{array}{l}\text { India } \\
\text { Total } \\
\end{array}$} \\
\hline & $\begin{array}{l}\text { Por razo- } \\
\text { nés médi- } \\
\text { cars }\end{array}$ & $\begin{array}{c}\text { Por razo- } \\
\text { nes perso } \\
\text { nales }\end{array}$ & Total & & $\begin{array}{l}\text { Por razo- } \\
\text { nes médi- } \\
\text { cas }\end{array}$ & $\begin{array}{c}\text { Por razo- } \\
\text { nes perso } \\
\text { nales }\end{array}$ & Total & & $\begin{array}{l}\text { Por razo- } \\
\text { nes médI- } \\
\text { cas }\end{array}$ & $\begin{array}{l}\text { Por razo- } \\
\text { nes perso } \\
\text { nales }\end{array}$ & Total & & & \\
\hline \multicolumn{15}{|l|}{ Doble $S(100 p)$} \\
\hline Talla A $(25 \mathrm{~m})$ & 12.0 & 6.1 & 28.1 & $19 \cdot 9$ & 11.5 & 6.1 & .17 .6 & 11.5 & & & & & & \\
\hline $\begin{array}{l}\text { Talla B }(31 \mathrm{~mm}) \\
\text { Talla C }(30 \mathrm{~mm})\end{array}$ & $\begin{array}{l}14.5 \\
12.5\end{array}$ & 3.4 & $\begin{array}{l}17.9 \\
14.7\end{array}$ & $\begin{array}{l}22.3 \\
42.7\end{array}$ & & & & & & & & & 5.9 & $(+, 0)$ \\
\hline Talla D $(27.5)$ & 14.2 & 3.1 & $17 \cdot 3$ & $\begin{array}{l}42.1 \\
28.0\end{array}$ & 7.2 & 3.6 & 10.8 & 7.7 & & & & $(5.0)$ & & \\
\hline \multicolumn{15}{|l|}{ Espiral } \\
\hline Pequeña & 23.3 & 10.2 & $23.5\}$ & \multirow[t]{2}{*}{26.6} & 12.1 & 6.3 & 18.4 & & & & & & ) & \multirow{2}{*}{$(7.0)$} \\
\hline Grande & 2.2 .9 & 4.8 & $26.7)$ & & 16.1 & 5.6 & 21.7 & & & & & & & \\
\hline \multicolumn{15}{|l|}{ Nudo (bow) } \\
\hline $\begin{array}{l}\text { Fequeño } \\
\text { Grande }\end{array}$ & $\begin{array}{l}12.4 \\
14.3\end{array}$ & $\begin{array}{l}4.7 \\
2.6\end{array}$ & $\begin{array}{l}17.2 \\
26.9\end{array}$ & & & & & & & & & & & \\
\hline Anillo de acero & 9.7 & 3.0 & 12.7 & & & & & & & & & & & \\
\hline Anillo de plástico & & & & & & & & & 6.3 & 2.I. & 8.4 & & & \\
\hline
\end{tabular}

Fuentes: mismas citadas en el cuadro 2; India, misma que Hong Kong. 
el primer año. Además, C. Tietze [20] observa que este modelo es la causa del mayor número de perforaciones del útero.

Según R. E. Hall [22], que ha hecho un estudio de las ventajas de los diferentes modelos de dispositivos intrauterinos, las tasas de expulsión y de efectos secundarios de la espiral son tan elevadas que esta forma deberá sin duda ser abandonada en el futuro. Llega a la misma conclusión respecto al pequeño nudo en razón de la elevada tasa de fracasos, mientras que el modelo doble S (loop) de talla grande $(31 \mathrm{~mm})$ es el que prefiere. Es similar la opinión de C. Tietze [20], quien recomienda el modelo de talla grande o mediana, así como la de los experimentadores de Formosa [17], quienes habiendo adoptado al comenzar el dispositivo de talla pequeña, prefieren utilizar en la actualidad modelos más grandes.

La retención del dispositivo intrauterino, sin ser perfecta, es sin embargo importante. Si toma uno en cuenta el total de fracasos, expulsiones y extracciones por razones médicas o personales, las tasas de retención calculadas por $\mathrm{C}$. Tietze [23] en un grupo de mujeres que pudieron ser estudiadas durante cuatro años en Estados Unidos son las siguientes (según un método de cálculo semejante al de la tabla de sobrevivientes ya indicado):

Tasa por 100 primeras

inserciones, sin con- Tasa por 100 inserciosiderar reinserciones nes, teniendo en cuen-

(todas las formas de ta reinserciones dispositivos)

(doble $S$, talla $D$ ) a
Al final del primer año
69.4
78.5
Al final del 2o, año
59.4
66.6
Al final del 3er. año
50.0
58.5
Al final del 40 . año
51.6

a Esta forma de dispositivo ha merecido las preferencias de los experimentadores, por su baja tasa de expulsión.

En Puerto Rico, la tasa de retención es de $83 \%$ al final del primer año, es decir, poco diferente a la de Estados Unidos. En Formosa, esta proporción no llega sino al 56.2\% para el dispositivo doble $\mathrm{S}$, talla $\mathrm{D}$, por razón de las numerosas extracciones de que se ha hecho mención. En Santiago de Chile es de $71.6 \%$ para el anillo de nyton. En Corea del Sur es de $76.2 \%$ para la doble S de talla A y de $89 \%$ para la talla D. En Túnez [11], de 75.2\% teniendo en cuenta las reinserciones. En Paquistán [11], de 78.6\% incluidas las reinserciones y de $71.3 \%$ sin reinserciones. En India [11], en Nueva Delhi, oscila entre el 78.0 y el $85.4 \%$ tomando en cuenta las reinserciones.

Puede en consecuencia afirmarse, grosso modo, que en el estado actual de la técnica y en las circunstancias más favorables, el dispositivo intrauterino parece permanecer durante un año entero por lo menos en tres de cada cuatro mujeres, tanto en los Estados Unidos 
como en los diversos países del Tercer Mundo que se han prestado a estos experimentos. Al final del tercer año esta proporción excede con frecuencia del $50 \%$.

Aun cuando la retención no sea perfecta, debe reconocerse, sin embargo, que los contraceptivos intrauterinos pueden permitir una esterilización casi absoluta durante períodos bastante largos y en proporciones elevadas de mujeres. Esto no se podría esperar antes de mucho tiempo con los procedimientos tradicionales, sobre todo en los países subdesarrollados. ${ }^{6}$ Falta saber si esta conclusión optimista seguirá siendo válida una vez que el procedimiento se difunda en gran escala.

La influencia de la edad de la mujer y el número de partos es considerable, tanto en los fracasos como en las expulsiones o extracciones del dispositivo intrauterino. Las proporciones de expulsión o de retiro por causa médica disminuyen muy marcadamente con la edad de la mujer y por lo tanto también con el número de hijos que ella haya podido tener, en tanto que las proporciones de fracasos varían en sentido inverso. Este fenómeno se muestra en los cuadros 5, 6 y 7 , en que se reproducen los datos de países que han proporcionado informaciones sobre el particular, esto es, Estados Unidos, Formosa y Chile.

En Estados Unidos, la tasa de expulsión del dispositivo entre mujeres de 15 a 24 años que han tenido 1 ó 2 niños, después de un año (dispositivo de talla $D, 31 \mathrm{~mm}$ ) se eleva a $7.3 \%$, en tanto que en las mujeres de 35 a 49 años que han tenido por lo menos 5 hijos, esta proporción es apenas de $1.2 \%$.

En Formosa la tasa, según las observaciones basadas en toda clase de causas, después de un año es de $54.9 \%$ en las mujeres de 13 a 24 años de edad, y de $23.3 \%$ en las de más de 35 años. La tasa es de $66.8 \%$ en las mujeres que han tenido uno o dos embarazos $\mathrm{y}$ de $23.5 \%$ en aquellas que han tenido por lo menos 6 embarazos. Diversas observaciones en la India y en Tailandia [11] confirman estas conclusiones.

Es explicable que las tasas de extracción sean elevadas en las mujeres que han tenido pocos hijos y son todavía muy jóvenes: cualquier molestia las inclina fácilmente a no aceptar el contraceptivo, tanto más que los efectos secundarios son de gran frecuencia, como se verá más adelante. No es igual tratándose de las tasas de expulsión o de fracaso, que al parecer dependen poco de la voluntad de la portadora y encuentran sobre todo explicaciones en la fisiología o en un mal ajuste del dispositivo intrauterino a la cavidad uterina.

Se ha hecho en Formosa la verificación siguiente: las tasas de baja por todas las causas, después de un año, aumentan en el número de abortos provocados (el cálculo ha eliminado el efecto de la edad o del número de partos). Según R. Potter [25], la tolerancia del útero a los contraceptivos disminuye con los abortos realizados.

6. B. M. Lee y J. Isbister [24], colocándose en ciertas hipótesis sugeridas por las observaciones de Corea del Sur (tasa de fecundidad, tasa de aceptación según la edad, tasa de expulsión, etc.), calculan que cada dispositivo insertado evitará en promedio un nacimiento en cinco años. 


\section{Cuadro 5}

Estados Unidos: Tasas de baja de ObSERvación después de UN aÑo, Según la edad de La MUJER Y EL NÚMERO DE PARTOS

(Dispositivos en forma de doble $\mathrm{S}$, de $31 \mathrm{~mm}$; t tasas netas por 100 observaciones)

\begin{tabular}{|c|c|c|c|c|c|c|c|c|c|c|c|c|}
\hline & \multicolumn{12}{|c|}{ Edad de la mujer } \\
\hline & \multicolumn{3}{|c|}{$15-24$ años } & \multicolumn{3}{|c|}{$25-29$ años } & \multicolumn{3}{|c|}{$30-34$ años } & \multicolumn{3}{|c|}{$35-49$ años } \\
\hline & $\begin{array}{l}\text { I a } 2 \\
\text { niños }\end{array}$ & $\begin{array}{l}3 \text { a } 4 \\
\text { niños }\end{array}$ & $\begin{array}{l}5 \mathrm{y} \\
\text { más }\end{array}$ & $\begin{array}{l}1 \text { a } 2 \\
\text { niños }\end{array}$ & $\begin{array}{l}3 \text { a. } 4 \\
\text { niños }\end{array}$ & $\begin{array}{l}5 y \\
\text { más }\end{array}$ & $\begin{array}{l}\text { 1. } 2 \\
\text { niños }\end{array}$ & $\begin{array}{l}3 \text { a } 4 \\
\text { niños }\end{array}$ & $\begin{array}{l}5, y \\
\text { más }\end{array}$ & $\begin{array}{l}\text { I a } 2 \\
\text { niños }\end{array}$ & $\begin{array}{l}3 \text { a } 4 \\
\text { niños }\end{array}$ & $\begin{array}{l}5 y \\
\text { más }\end{array}$ \\
\hline Fracasos & 3.2 & 2.7 & 2.5 & 2.3 & 2.6 & 3.3 & 1.2 & 1.9 & 1.2 & 0.6 & 1.0 & 2.0 \\
\hline Expulsiones & 7.3 & 6.5 & $5: 7$ & 5.3 & 3.7 & 2.0 & 3.2 & 2.5 & 2.5 & 2.7 & 1.5 & 1.2 \\
\hline Extracciones & 17.8 & 12.9 & 13.5 & 17.2 & 14.2 & 10.2 & 15.4 & 11.8 & 14.5 & 15.7 & 12.8 & 9.2 \\
\hline $\begin{array}{l}\text { Rodavía en observación al fin. } \\
\text { del primer año }\end{array}$ & 71.7 & $77 \cdot 9$ & 78.3 & 75.2 & 79.5 & 84.5 & 80.2 & 83.8 & 81.8 & 81.0 & 84.7 & 87.6 \\
\hline
\end{tabular}




\section{Cuadro 6}

Formosa: TASAS de baja de OBSERVACIÓN POR CADA 100 PRIMERAS INSERCIONES AL FIN DEL PRIMERO Y EL SEGUNDO AÑOS

(Dispositivos en forma de doble S)

\begin{tabular}{|c|c|c|c|c|c|}
\hline $\begin{array}{l}\text { Edad en la } \\
\text { primera in } \\
\text { serción }\end{array}$ & $\begin{array}{l}\text { Fin del } \\
\text { primer } \\
\text { año }\end{array}$ & $\begin{array}{l}\text { Fin del } \\
\text { segundo } \\
\text { año }\end{array}$ & $\begin{array}{l}\text { lúmero de nam } \\
\text { cidos vivos } \\
\text { o nacidos an- } \\
\text { tes de la pri } \\
\text { mera inserción }\end{array}$ & $\begin{array}{l}\text { Fin del } \\
\text { primer } \\
\text { año }\end{array}$ & $\begin{array}{l}\text { Fin del } \\
\text { segundo } \\
\text { año }\end{array}$ \\
\hline $13-2^{4}+$ años & $54.9 \pm 1.9$ & $75.8-2.2$ & $0-1$ & $65.8+3.6$ & 90.8 \\
\hline 25-29 años & $40.1 \pm 1.2$ & $60.5 \pm 1.5$ & 2 & $53.3 \pm 2.0$ & 72.4 \\
\hline $30-34$ años & $28.6 \pm 1.1$ & $44.6 \pm 1.5$ & 3 & $41.3-1.5$ & $61.7^{+}$ \\
\hline \multirow[t]{3}{*}{$\begin{array}{l}35 \text { años y } \\
\text { más }\end{array}$} & $23.3^{+}-1.1$ & $35.4 \pm 1.6$ & 4 & $32.9 \pm 1.4$ & $51.9 \pm 1.9$ \\
\hline & & & 5 & $28.6 \pm 1.4$ & $43.8 \pm 2.0$ \\
\hline & & & $6-17$ & $23.5 \pm 1.0$ & $36.5^{+}-1.5$ \\
\hline
\end{tabular}

Cuadro 7

Chile: TASAS DE FRaCaso Y DE eXtracción DEL dispositivo (anillo DE NYLóN) POR CAUSAS MÉDICAS, SEGÚN EL NÚMERO DE PARTOS

(Tasas por 100 observaciones en el primer año)

\begin{tabular}{|c|c|c|c|c|c|}
\hline \multirow[b]{2}{*}{ Número de partos } & \multirow{2}{*}{$\begin{array}{l}\text { Múmero } \\
\text { de ob- } \\
\text { serva- } \\
\text { ciones }\end{array}$} & \multicolumn{2}{|c|}{ Expulsiones } & \multicolumn{2}{|c|}{ Extracciones } \\
\hline & & $\begin{array}{l}\text { jumero } \\
\text { de ca- } \\
\text { sos }\end{array}$ & Tesas & $\begin{array}{l}\text { Numero } \\
\text { de ca- } \\
\text { sos }\end{array}$ & Tasas \\
\hline 0 & 2 & 0 & - & 1 & - \\
\hline 1 & 63 & 13 & - & 3 & - \\
\hline 2 & 287 & 69. & $26.4 \pm 2.9$ & 23 & $8.5 \pm 2.0$ \\
\hline 3 & 617 & 110 & $18.9 \pm 1.7$ & 39 & $6.3 \pm 1.1$ \\
\hline $4-5$ & 1044 & 161 & $16.8 \pm 1.2$ & 83 & $6.7 \pm 0.9$ \\
\hline $\begin{array}{l}6-7 \\
8 \text { y más }\end{array}$ & $\begin{array}{l}545 \\
397\end{array}$ & $\begin{array}{l}72 \\
38\end{array}$ & $\begin{array}{l}14.0 \pm 1.6 \\
10.1 \pm 1.6\end{array}$ & $\begin{array}{l}38 \\
19\end{array}$ & $\begin{array}{l}6.0 \pm 1.8 \\
3.7 \pm 1.1\end{array}$ \\
\hline Indeterminados & 45 & 5 & - & 1 & - \\
\hline Total & 3000 & 468 & $27.2 \div 0.8$ & 207 & $6.3 \pm 0.5$ \\
\hline
\end{tabular}


La frecuencia de los efectos secundarios del dispositivo uterino es bastante elevada. R. E. Hall [22] ha comparado los efectos secundarios de las diferentes formas del dispositivo intrauterino y señala en los experimentos norteamericanos - con relación al dispositivo que parece responder a condiciones óptimas, es decir, el que tiene la forma de doble $\mathrm{S}$ y es de talla grande- una proporción de efectos secundarios, de embarazos no deseados y de expulsiones al final del primer año, de $36.9 \% .^{7}$ La proporción de inserciones que acarrean efectos secundarios suficientemente graves para justificar en todo caso la extracción del dispositivo, es de $8.3 \%$. Los efectos secundarios más frecuentes son las pérdidas de sangre y las reacciones dolorosas, que motivan desde luego las dos terceras partes de las extracciones por razones médicas.

C. Tietze [26] indica una tasa que varía de 2 a $4 \%$ sólo por lo que hace a las inflamaciones pelvianas en los Estados Unidos, que ciertamente no aparecen sino al cabo de varios meses. La mayor parte de las enfermedades pueden ser tratadas eficazmente por los antibióticos sin que sea necesario retirar el dispositivo.

Es interesante hacer notar que hasta ahora no se ha comprobado ningún efecto secundario sobre la lactancia, cuya importancia es tan grande en los países subdesarrollados, en tanto que la píldora parece interrumpirla o modificarla, siendo influida la lactación desfavorablemente cuando se absorben fuertes dosis. ${ }^{8}$ Este inconveniente de la píldora no se presenta más que en las mujeres en las cuales la lactancia no es acompañada de alguna esterilidad temporal, lo que no ha sido establecido siempre de modo seguro.

La proporción de abandono de la contracepción parece ser más elevada en Estados Unidos tratándose del dispositivo intrauterino que de la píldora. El cuadro 8 muestra, en un grupo de 14365 mujeres que en Chicago han empleado la píldora (Enovid), los porcientos de continuación del método [27] comparados con las tasas de retención del dispositivo en el grupo de mujeres estudiadas, bajo las encuestas del National Committee on Maternal Health, por 38 investigadores $o$ instituciones diferentes [19].

Queda por saber en qué medida los dos grupos de mujeres son comparables. Parece que las mujeres que han adoptado la píldora son más jóvenes que las que han escogido el dispositivo intrauterino como medio de limitación de nacimientos; pero la diferencia no deja de llamar la atención. Si del 70 al $83 \%$ de las mujeres que han adoptado la píldora continúa utilizándola después de dos años y medio, en cambio las tasas de continuación no son más que del 50 al $60 \%$ en las que han escogido el dispositivo intrauterino. Muchas hipótesis podrían sugerirse para explicar estas diferencias: efectos secundarios más frecuentes con el dispositivo que con la píldora, extracciones y abandono, tasas de expulsión no despreciables (como hemos visto en el caso del dispositivo), etc.

7 Se trata de una tasa por 100 años-mujer calculada según el método de la tabla de sobrevivientes de R. G. Potter indicada más arriba.

8 Algunos autores suponen una filtración de progestativos en la leche materna, lo que tendría consecuencias en la salud del niño; pero no se ha verificado esta hipótesis. 


\section{Cuadro 8}

Estados Unidos: Comparación de CONTINUACIón EN EL USO de contraceptivos, Chicago, 1965

\begin{tabular}{|c|c|c|c|}
\hline & \multicolumn{2}{|c|}{$\begin{array}{l}\text { Proporción de con- } \\
\text { tinuación de la } \\
\text { píldora }(\%)\end{array}$} & \multirow[t]{2}{*}{$\begin{array}{l}\text { Proporción de re- } \\
\text { tención del dis- } \\
\text { positivo }(\%) \text { c }\end{array}$} \\
\hline & $\mathrm{A}$ a & $\mathrm{B}$ b/ & \\
\hline \multicolumn{4}{|l|}{ A] final del: } \\
\hline Primer trimestre & 94.4 & 87.8 & 85.2 \\
\hline Segundo trimestre & 92.1 & 83.1 & 78.5 \\
\hline Tercer trimestre & 90.8 & 80.4 & 73.4 \\
\hline Cuarto trimestre (un año) & 89.5 & 77.4 & 69.4 \\
\hline Sexto trimesire & 87.3 & 73.9 & 62.4 \\
\hline Octavo trimestre (dos & & & \\
\hline años) : & 85.0 & 71.7 & 59.4 \\
\hline Décimo trimestre & 83.1 & 70.2 & 54.6 \\
\hline
\end{tabular}

a No se ha tenido en cuenta en el cálculo sino a las mujeres que han cesado de usar la píldora por razones consideradas graves (embarazos no deseados, náusea, aumento de peso, etc.). Estas proporciones representan por lo tanto límites máximos. Han sido calculfidas según el método de R. G. Potter.

b Estas cifras toman en consideración a las mujeres que han dejado de usar la píldora, a la vez que por razones graves, por otras consideradas no graves, o que han abandonado la encuesta (deseos de un nacimiento, preferencia por otro tipo de contraceptivo oral, etc.). Estas tasas representan límites considerados mínimos por el mismo autor de los cálculos.

c Proporciones por 100 primeras inserciones, sin tener en cuenta las reinserciones, respecto a todas las formas de dispositivos intrauterinos. Estas proporciones son un poco más elevadas si se tiene en cuenta solamente el dispositivo doble $\mathrm{S}$ de talla $\mathrm{D}$, que parece estar convirtiéndose en el modelo más corriente, y las reinserciones.

Difícilmente se pueden comparar todavía los efectos secundarios $y$ las contraindicaciones de los progestativos y de los contraceptivos intrauterinos. En los cuadros 9 y 10 se indican las listas de los efectos secundarios y las contraindicaciones para los diferentes procedimientos de limitación de nacimientos, recogidos por A. L. Southam [28].9

Se puede ver que estas listas son más largas con respecto a los procedimientos nuevos que a los tradicionales, y entre los primeros con relación a los progestativos orales que a los contraceptivos intrauterinos. Sin embargo, es difícil hacer una comparación rigurosa, porque exigiría un estudio profundo de todos los aspectos médicos.

En lo que concierne al dispositivo intrauterino, las mujeres que se proveen del mismo cuando ya están encinta, o que son fecundadas después de la colocación del dispositivo, abortan más frecuentemente

${ }^{9}$ En dos folletos de la Organización Mundial de la Salud se encontrarán informes sobre los efectos de los dispositivos intrauterinos y los gestágenos orales [29]. 


\section{Cuadro 9}

EfEctos SECUNDARIos asociados a difERENTES MÉTOdos CONTRACEPTIVos a

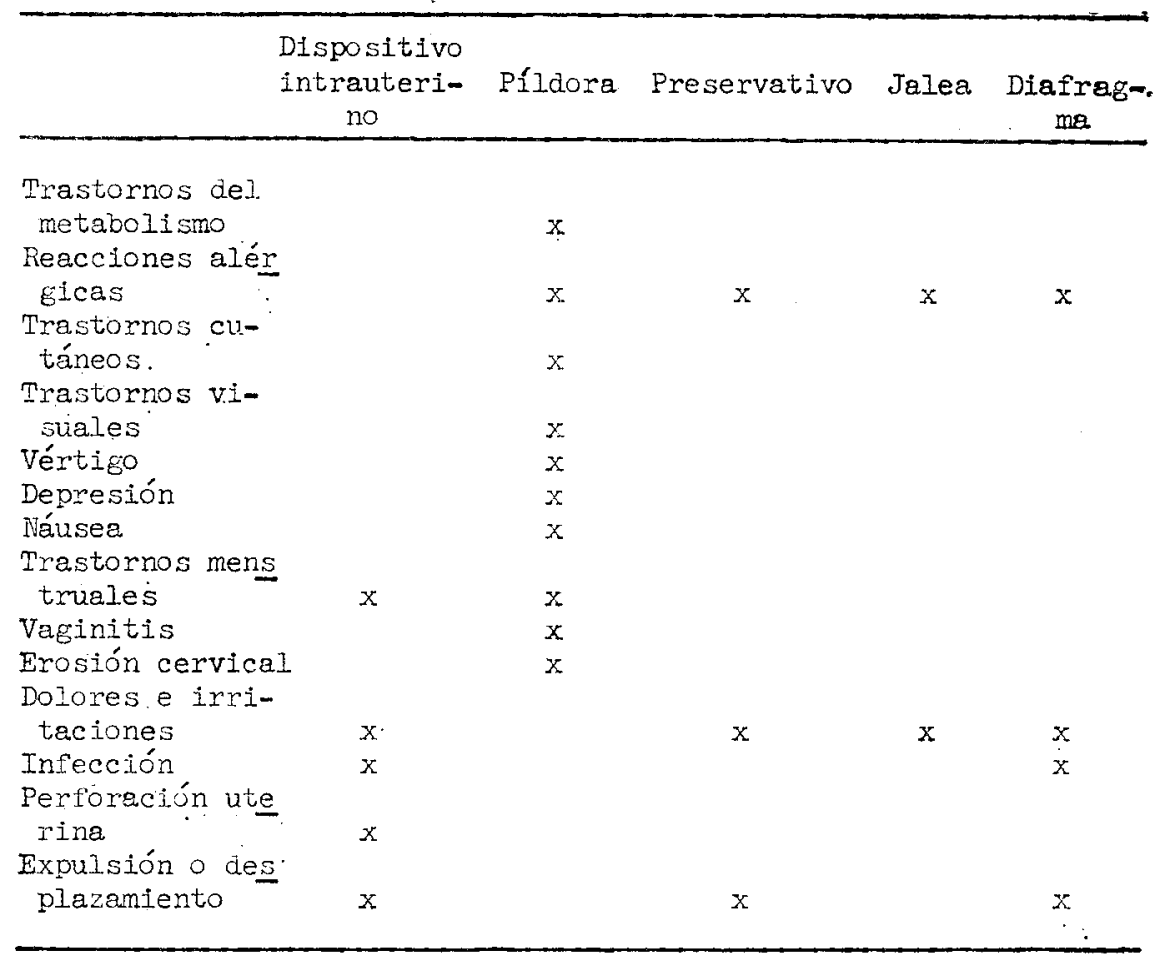

Fuente: [28].

a Conviene agregar a esta tabla, entre los efectos secundarios encontrados en la píldora, Ia interrupción o la modificación eventual de la lactancia, trastorno que no se encuentra en los otros métodos contraceptivos.

que las que conciben después de haber expulsado el dispositivo sin darse cuenta [29]. La Organización Mundial de la Salud [29] hace ver, en lo que concierne a los progestativos orales, que la recuperación de la función ovárica después de utilizar prolongadamente el producto puede durar cierto tiempo (uno a tres ciclos y a veces más).

Debido a que no se dispone de información numérica, es difícil tener una idea precisa de la importancia relativa de los efectos secundarios de los diferentes procedimientos. El problema es, sin duda, complicado.

Desde el punto de vista cuantitativo es muy difícil responder a la cuestión de saber si estos efectos secundarios son más frecuentes con un procedimiento que con otro. Los factores síquicos pueden jugar, en efecto, un papel evidente en la apreciación de los efectos secundarios, sobre todo aquellos que se relacionan con los progestativos orales; ciertas mujeres tienen tendencia a culpar a la droga de todos los malestares que resientan durante el período de la experimen- 
Cuadro 10

CONTRAINDICACIONES EN LOS DIFERENTES MÉTODOS CONTRACEPTIVOS

\begin{tabular}{|c|c|c|c|c|}
\hline & $\begin{array}{l}\text { Dispositivo } \\
\text { intrauteri- } \\
\text { no }\end{array}$ & Píldora & Ogino & $\begin{array}{c}\text { Diafrag- } \\
\text { ma }\end{array}$ \\
\hline $\begin{array}{l}\text { Tromboflebitis } \\
\text { Enbolia } \\
\text { Accidentes cardlovasculares } \\
\text { Cardiopatias } \\
\text { Males renales } \\
\text { Trastornos hepáticos } \\
\text { Trastornos de la vista } \\
\text { Trastornos tiroideos } \\
\text { Hemorragias } \\
\text { Ciclos irregulares } \\
\text { Cáncer del seno } \\
\text { Cáncer del aparato genital } \\
\text { Fibroma uterino } \\
\text { Infección pelviana } \\
\text { inulipariedad } \\
\text { Expulsiones repetidas } \\
\text { Defectos de la morfología } \\
\text { pelviana }\end{array}$ & $\begin{array}{l}x \\
? \\
x \\
x \\
x\end{array}$ & $\begin{array}{l}x \\
x \\
x \\
x \\
x \\
x \\
x \\
x \\
x \\
x \\
? \\
?\end{array}$ & $\mathrm{x}$ & 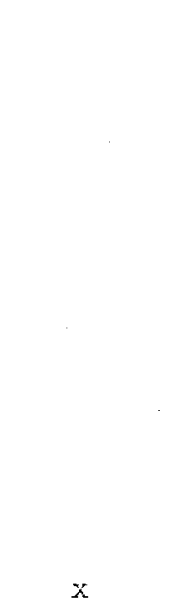 \\
\hline
\end{tabular}

Fuente: [28].

tación [30]. En cuanto a los contraceptivos intrauterinos, llama la atención la tasa de $36.9 \%$ de efectos secundarios (benignos o graves) dada por Hall [22].

Los efectos secundarios tardios de los nuevos contraceptivos casi se ignoran. Los efectos secundarios que se han examinado hasta aquí se limitan a los inmediatos; los a largo plazo son todavía casi desconocidos, y en realidad los temores suscitados por el uso de los contraceptivos nuevos tienen que ver más con los efectos a largo plazo que con los inmediatos o los que puedan surgir después de un corto número de años. En particular, hay que reconocer que las hipótesis sombrías que se han propuesto como ciertas, como la de una acción cancerógena, no pueden ser descartadas todavía de manera formal, ya que los periodos de observación son aún demasiado cortos.

Numerosos médicos, sobre todo en Europa, son renuentes a la difusión de los contraceptivos intrauterinos porque piensan que la introducción de un cuerpo extraño en el útero, localización de una importante proporción de los cánceres en la mujer, no puede ser aceptada sin que se corran en el futuro los riesgos inherentes a toda irritación crónica. El cáncer tiene una larga vida oculta, se dice, y se 
puede temer que simples irritaciones, en apariencia sin gravedad, degeneren en él.10

Este mismo espectro del cáncer ha sido discutido a propósito de los progestativos orales, ya que el período de la incubación de un cáncer determinado por un agente químico puede alcanzar numerosos años. Además, otros desórdenes fisiológicos debidos a la administración continua de un medicamento destinado a inhibir, por una acción hormonal, la función ovárica, podrían aparecer a la larga; por ejemplo, un alargamiento del período de reproducción.

No es imposible que, haciendo alusión a estas incertidumbres, se tenga presente en la memoria el drama de la talidomida, medicamento utilizado como sedante destinado a calmar los vómitos del embarazo, y de hecho inofensivo para el adulto, mientras que, administrado a una mujer encinta, es capaz de producir efectos desastrosos en el niño que lleva en el vientre.

Las conclusiones de los expertos franceses [30] indican que las incertidumbres actuales sobre los efectos secundarios de los contraceptivos orales no sobrepasan a aquellos encontrados en numerosos medicamentos de uso corriente y que ningún caso de cáncer puede ser atribuido por el momento, de manera formal, a los contraceptivos, sea que se trate del dispositivo intrauterino o de la píldora. Se puede afirmar ya que el empleo de un contraceptivo intrauterino no está asociado a ninguna transformación neoplásica rápida.

Pero, ¿el planteamiento en estos términos es correcto? Porque lo que importa es el riesgo involucrado por el empleo continuo de este procedimiento durante largos períodos, que pueden alcanzar hasta 25 ó 30 años, caso con el que se tropieza muy rara vez tratándose de medicamentos usuales a escala de una población entera. Hasta la toma de un comprimido de aspirina presenta inconvenientes serios para la salud cuando esto se hace cotidianamente. Casi no hay duda de que se afrontarán nuevos problemas de salud pública cuando el dispositivo intrauterino se difunda en gran escala. Pero nada indica por el momento que no se les pueda llegar a dominar.

La honradez exige que en todos estos planes se distingan las hipótesis de las certidumbres. Todo investigador conoce la dificultad que existe para establecer un hecho preciso y necesita por ello proceder lentamente, no admitiendo sino lo que ha sido verificado, y esto exige siempre tiempo. Muchos investigadores, en plan de hombres de acción, creen ir más de prisa. Por lo menos, conviene que reconozcan el carácter especulativo de sus empresas. Si en las ciencias exactas se prueba sobre la marcha el avance del conocimiento, esto no puede aceptarse sino con infinitamente más prudencia en las ciencias humanas, sobre todo, cuando presionados por el tiempo, los experimentos se realizan en centenas de millares de mujeres, y aun en millones, y se ha pasado ya bastante a la fase de la difusión, sin esperar todavía los resultados de las investigaciones experimentales.

Se explica uno en estas condiciones por qué muchos médicos se rehusan a intervenir, y su reticencia se apoya en dos puntos. Desde

10 En el caso del dispositivo intrauterino, no podria ocurrir una cancerogénesis eventual más que local, es decir, alcanzar el endómetro o el epitelio del cuello. 
luego, la idea de intervenir para modificar la fisiología; en este caso disminuir una función normal, sin que haya un deterioro en el estado de la salud, responde a una nueva concepción del papel del médico en la sociedad, que muchos rechazan. Corregir la fisiología natural les parece una idea diabólica y peligrosa cuando no son fácilmente comprensibles para la persona los hechos patológicos. Por otra parte, numerosos médicos rehusan interesarse en los aspectos sociológicos planteados por los problemas de la salud entendidos en un sentido tan amplio. Porque, en realidad, el individuo no está enfermo, sino que la sociedad sufre las consecuencias de su propia exuberancia. Pero hay que reconocer que estas reservas son cada día menos tajantes y se nota una evolución y una ampliación de las ideas en la apreciación de lo normal y de lo patológico.

\section{LOS PROBLEMAS DE DIFUSIÓN}

A partir de algunas sencillas observaciones se intentará clasificar los factores favorables o desfavorables a la difusión del dispositivo intrauterino en el Tercer Mundo.

Mientras que en los países subdesarrollados la elección entre los diversos métodos contraceptivos se deja a la población, ella se ha dirigido hasta ahora más hacia el dispositivo que hacia los métodos tradicionales o la píldora. En un país subdesarrollado se ofrecen diferentes métodos contraceptivos al gusto del consumidor (todos los procedimientos son de alguna manera expuestos, explicados y propuestos); pero el dispositivo ha sido sin duda el de más éxito por el momento, y el que ha sido aplicado con más fidelidad. De este modo, en Tailandia, en la zona de experimentación de la zona rural de Photharam, accesible sólo por barco, y donde la contracepción era hasta entonces desconocida, el $77 \%$ de las mujeres que manifestaron el deseo de limitar sus concepciones escogieron el dispositivo, $13 \%$ la píldora, y $9 \%$ el preservativo masculino [31]. En una encuesta hecha en Puerto Rico [32] entre noviembre de 1961 y noviembre de 1963, se seleccionaron dos grupos de mujeres: el primero, de 660, escogió el progestativo oral, y el otro, de 608, prefirió el contraceptivo intrauterino. Aunque los dos grupos de muestra no sean perfectamente comparables (las que prefirieron el dispositivo son las mujeres de avanzada edad o que han tenido bastantes hijos, y las que eligieron la píldora se encuentran entre las mujeres de medios socioeconómicos más favorecidos), llaman la atención los promedios de continuación de métodos después de dos años: $70 \%$ con el dispositivo intrauterino y $48 \%$ con la píldora. Parece que se observa en todas partes un fenómeno inverso al anotado en páginas anteriores respecto a Estados Unidos. Pero todo esto requiere confirmación. ${ }^{11}$

En Formosa [17], siguiendo siempre el principio del "gusto del consumidor", sólo el $2 \%$ de las mujeres se ha decidido por la píldora. Tras de siete meses, la tasa de continuación era de $80 \%$. Pero el $90 \%$

11 Todavía queda por saber en qué medida los experimentadores han influido a' las mujeres alabando más los méritos del dispositivo que de los otros procedimientos contraceptivos. 
: las mujeres había dejado de tomar la píldora al menos una vez en intervalo de los siete meses de la encuesta, a pesar de que el nivel Iltura! y social de este grupo de mujeres estaba considerado como lativamente elevado.

No se pueden descuidar los métodos tradicionales si se quiere obner una fuerte disminución de la natalidad en el Tercer Mundo. endo las proporciones de retención del dispositivo por el momento oderadas y la tolerancia a ese procedimiento aún imperfecta, se Izga necesario asociar, según lo sugieren J. F. Kanter [33] y R. Freedan [34], a la difusión del dispositivo la de otros procedimientos, si : desea obtener una disminución considerable de la natalidad en el ercer Mundo. El dispositivo, con todo y sus ventajas evidentes, y a particular su tasa mínima de fracaso, no es todavía el arma absolu1, la panacea capaz de barrer desde ahora con todos los otros meios de limitación de nacimientos. Esto aparece tanto más verdadero santo que el aparato médicosocial que exige una amplia difusión e los contraceptivos intrauterinos no podrá estar a la disposición de is países subdesarrollados antes de transcurrido mucho tiempo.

La India ha comprendido esto tan bien que prevé disminuir los naimientos en 1975 recurriendo a toda una gama de medios [35]:

Nacimientos evitados por un aumento de la edad de casamiento

Nacimientos evitados por la esterilización

Nacimientos evitados por el dispositivo intrauterino

Nacimientos evitados por el preservativo

1.9 millones

$2.1 \quad$,

$4.0 \quad$,

$1.0 \quad$,

9.0 millones

El Comité Especial de Evaluación de Programas de Planificación amiliar, creado en 1964 en la India [35] para ayudar al gobierno a leterminar un plan de acción, ha enviado equipos de observadores : los diversos estados para evaluar la aceptabilidad de los métodos. as recomendaciones del Comité se han inclinado no sólo por el dissositivo sino también por la esterilización y el preservativo masculino.

Igualmente, las informaciones procedentes de Paquistán y de Ceián muestran la importancia que los dirigentes dan a los medios tradiionales y a la esterilización.

Los obstáculos a la difusión del dispositivo en los países subdesarrollados dependen por el momento de la influencia del aparato nédicosocial. Para muchos observadores, la clave de una difusión en il Tercer Mundo de la contracepción fundada en el dispositivo reside in la organización de un programa nuevo de acción que deberá apoyarse en un aparato médicosocial de por sí bastante insuficiente para encarar los problemas de la salud y que puede dañarla en la medida in que este nuevo programa pueda afectarla.

Las dificultades son, en efecto, numerosas. Se trata no sólo de colozar contraceptivos intrauterinos, sino también, y sobre todo, de hacer exámenes clínicos que permitan identificar los casos en que se presen- 
ten contraindicaciones para excluir en éstos la aplicación; proceder en seguida a exámenes suficientemente repetidos en las mujeres parć descubrir lo antes posible los casos de expulsión, hacer la reinserción, ${ }^{1:}$ y vigilar la aparición y evolución de efectos secundarios cuando se presenten. Todas estas actividades exigen personal médico y para médico del que a menudo se carece, puesto que en muchos países la sicología social exigiría que dicho personal fuera femenino, aunque ninguna encuesta sociológica haya esclarecido este punto con certeza

Todas estas dificultades se presentan de manera más aguda en el medio rural que en el urbano, ${ }^{13}$ en los países grandes como la India $\mathrm{y}$ en aquellos en que la población es numéricamente menos impor tante como Formosa,14 Corea del Sur o Puerto Rico, que resaltan entre los ejemplos de éxito reciente.

El principio mismo de la contracepción parece recibir una acogida favorable en casi todo el Tercer Mundo. La limitación de los nacimientos corresponde a una necesidad sentida en todas partes desde que la cuestión se propuso en forma abierta. El hecho mismo de poner a discusión la posibilidad de modificar voluntariamente la fecundidad constituye un poderoso estimulante hacia un cambio de comportamiento. Los testimonios que se pueden dar de esto abundan, como el de los ensayos contraceptivos intrauterinos hechos en Madurai, en la región de Madrás, India [35], donde se ha visto a los campesinos detener la ambulancia del hospital municipal para pedir el contraceptivo; o como el del hospital Lady Hardinge, de Nueva Delhi, donde las peticiones han sido tan abundantes que se ha tenido que impedir la entrada desde temprano, teniendo muchos que irse con las manos vacías. O como el éxito de la experimentación en la zona rural de Tailandia apenas accesible, antes citada [21], donde después de seis meses el $20 \%$ de las mujeres pidió el dispositivo mientras que antes no habían usado ningún método contraceptivo. Numerosas observaciones confirman que los dispositivos intrauterinos son igualmente aceptados en las zonas rurales que en las urbanas.

Ciertamente se pueden citar todavía muchas poblaciones donde proporciones importantes de las mujeres prefieren remitirse "a la voluntad de Dios". La fecundidad ha sido siempre santificada en todas las mitologías y en todas las religiones, apareciendo con frecuencia como una comunicación entre el hombre y lo sobrenatural, y guardará du-

1.2 Se calcula que con una "esperanza de vida" de 3.7 años del dispositivo, se necesitaría proceder todos los años, en una población de 1000 mujeres que lo desearan conservar de un modo permanente, a 270 reinserciones. Si la esperanza de vida fuera de 2.5 años, el número anual de reinserciones sería de 400 [11].

13 La carencia de médicos en el medio rural en los países subdesarrollados toma a veces cariz trágico. Se estima que en la India hay un médico por cada 5000 habitantes; en el estado de Madhya Pradesh, uno de los más rurales, la relación es de uno por cada 58000 habitantes. La "fuga de cerebros" en el medio médico es bien conocida. Así, en Punjab, de los 450 médicos diplomados cada año, se estima que 200 emigran para ejercer en otro lugar de la India o en el extranjero [35].

14 En el caso de Formosa, donde se estima que en 1963 el $7 \%$ de las mujeres casadas de 20 a 39 años llevaba dispositivo, esta práctica comenzó hace más de 20 años bajo la forma del anillo de Ota [17]. 
nte mucho tiempo todavía un lugar privilegiado en las sociedades ya cultura permanezca muy cerca de la naturaleza.

A veces las motivaciones son formuladas de manera más directa: ; mujeres prefieren, por ejemplo, no limitar deliberadamente sus cimientos con la esperanza de procurarse para la edad avanzada apoyo moral y material en los hijos que hayan traído al mundo.

Pero la coexistencia de estos dos tipos de motivaciones contrarias - hace en realidad más que traducir sentimientos ambivalentes que encuentran sobre otros muchos planos desde que los valores tradisnales entran en conflicto con los cambios que comienzan a producse en la sociedad, como lo ha mostrado claramente R. Freedan [34]. Esta ambivalencia explica los resultados en apariencia ntradictorios que se advierten en ciertas encuestas, como la que el aur llevó a cabo en Santiago de Chile en 1959 [36], donde el número eal de hijos está en estrecha correlación con el grado de religiosiid. Las mujeres cuya frecuencia de asistencia a los servicios religiosos a más fuerte se pronunciaban por un número ideal de hijos más evado, mientras que no se observó ninguna fecundidad diferencial al según la religiosidad. Las respuestas sobre el número ideal de jos son dictadas con frecuencia por cartabón, por opciones de carác$r$ moral o religioso o simplemente por la tradición, y pueden tener lación apenas mínima con el comportamiento efectivo. Más interentes parecen los análisis de las respuestas a las preguntas más rectas y menos ambiguas, como, por ejemplo: "¿Desea usted no ner más hijos?" En catorce países subdesarrollados donde la preinta se formuló, el porciento de respuestas positivas varió de 42 $93 \%$ en las mujeres que tienen tres hijos y de 69 a $95 \%$ en las que enen cuatro. Estas proporciones son con mucha frecuencia apenas feriores a las observadas en los Estados Unidos, donde han alcanIdo 62 y $81 \%$, respectivamente [37].

Hay ya poca duda de que la contracepción responde a un deseo asi universalmente compartido. $\mathrm{Y}$, en realidad, como bien lo muesan los resultados preliminares de siete encuestas comparativas sobre fecundidad en América Latina [38], las mujeres no tienen a menudo no escasos prejuicios desfavorables a la regulación de los nacimienss, aun siendo católicas. Este interés por la contracepción aparece por - regular muy pronto. Así, en las ciudades donde se han hecho is encuestas latinoamericanas, el porciento de las mujeres católicas ue declararon haber usado un método contraceptivo varió de 49 $93 \%$ antes del tercer embarazo. Pero los métodos empleados son oco eficaces - y sobre todo mal aplicados - siendo los más corrienss el lavado vaginal, la abstinencia periódica y el coito interrumido [39].

Las oportunidades de éxito de la contracepción en el Tercer Mundo on ya evidentes. No debería uno extrañarse del éxito encontrado por ts campañas de difusión del dispositivo, recibidas frecuentemente con livio por las mujeres. El procedimiento les parece, más que cualquier tro, como la solución a un problema que ellas no siempre se habían ropuesto claramente pero que pre-existía al menos en sus conciencias que ellas eran incapaces de resolver por sí mismas. 
En las políticas de gran envergadura que empiezan a ponerse el práctica, las oportunidades del dispositivo o de cualquier otro prc cedimiento que lo reemplace - las investigaciones de los laboratorio se orientan hacia procesos inmunológicos capaces de impedir la fecun dación [40]- están ya inexorablemente inscritas en el porvenir de Tercer Mundo. El crecimiento actual de la población y, todavía más el que se prevé en el futuro, necesitan naturalmente de un correctivo Tarde o temprano el cuerpo social todo entero deberá reaccionar es pontáneamente. Además, las barreras sicológicas, de las que se h: hecho mucho caso hasta ahora, se desmoronarán ante las ventaja: de un procedimiento reversible, que no implica molestia sexual, cuy: decisión deberá tomarse una sola vez (salvo el caso, ya se entiende de expulsión o de retiro, lo cual, según se ha visto, está lejos de ser la excepción), y que tiene una eficacia casi total. El procedimiento tien un mérito importante en el que quizás no se ha hecho hasta aqu: suficiente hincapié, a saber, que se deja a las mujeres el dominic de la reproducción y que se las sustrae de las consecuencias de le irresponsabilidad sexual de su compañero. Es éste sin duda uno de los factores de independencia más poderosos que pueden encontrar las mu. jeres con respecto a sus cónyuges y a la sociedad en general, y en este sentido el procedimiento puede ser calificado de verdaderamente re. volucionario.

Todas estas ventajas hacen que este "injerto de civilización" pueda ser implantado sin que sea necesario esperar cambios paralelos en el medio social. Esto parece tanto más notable cuanto que hasta ahora no se ha observado disminución de la fecundidad sin que la hayan precedido modificaciones en el cuadro sociocultural, capaces de favorecer el surgimiento de motivaciones en favor de la limitación de los nacimientos. La idea a menudo repetida - y con razón, pues da buena cuenta de la realidad- según la cual los motivos son a este respecto más importantes que los medios, parece que debe abandonarse. Estamos, en efecto, ahora en posesión de medios susceptibles de ser adoptados en ausencia de motivaciones fuertes.

Todo esto no quiere decir que el problema esté totalmente resuelto. Las dificultades, que no deben subestimarse, dependen sobre todo, como se ha visto, de los riesgos a largo plazo con respecto a la salud. Dependen también de la necesidad de dar nuevas responsabilidades a la estructura médicosocial, de por sí insuficientes para afrontar actualmente el problema de la salud. Se exige de ésta no sólo la colocación de los dispositivos, tomando todas las precauciones del caso cuando se presenten contraindicaciones, sino también la vigilancia de posibles expulsiones, la práctica de extracciones y la corrección de efectos secundarios cuya frecuencia - ya digna de tomarse en cuenta en una población como la de Estados Unidos- amenaza ser más fuerte en donde las condiciones de higiene disten de ser tan buenas.

Los peligros en el plano sociológico pueden ciertamente mencionarse en la medida en que esta "revolución del dispositivo" pueda crear desórdenes en estas sociedades. ¿No ocurriría, por ejemplo, que la noción de responsabilidad en el terreno de lo sexual, ya muy atenuada, desapareciera totalmente? Pero esta amenaza pertenece por entero 
al terreno de las hipótesis y aparece más como una consecuencia posible que como una causa de fracaso de las políticas en curso.

Se puede sostener que aun si mañana se debiera abandonar por razones técnicas la difusión del dispositivo, las campañas actuales de propaganda habrían tenido al menos el mérito de suscitar un movimiento colectivo de evolución de actitudes que con razón puede estimarse irreversible. Se ha dicho con frecuencia que una de las fuentes principales de resistencia al cambio es el miedo de apartarse de las normas. Estas campañas favorecen precisamente una modificación de los hábitos de los grupos más que de los individuos aislados, proponiendo francamente, a menudo por medio de discusiones, un problema que dejaba hasta ahora a los individuos presa de sus prejuicios, vacilaciones y veleidades. A fin de cuentas, es quizás esta transformación de actitudes lo que más importa.

\section{CONSECUENCIAS DESDE EL PUNTO DE VISTA DE LA INVESTIGACIÓN}

¿Qué relación existe entre la acción en curso y la investigación demográfica fundamental? Un verdadero problema de carácter epistemológico se plantea en la medida en que las políticas recientes, al absorber la atención de muchos investigadores, corran el riesgo de volver a plantear el problema del valor y el alcance de toda la investigación demográfica.

Nuevas investigaciones en el plano sicológico son necesarias ahora más que nunca. Nos encontramos empeñados, por estas políticas de población, en lo que se llama investigaciones activas, es decir, en las que la investigación es solidaria de la intervención. Esta reciprocidad entre conocimiento y acción debería normalmente incitar al investigador a un análisis cuidadoso de las situaciones concretas a las que él desea llevar la acción. Parece que las encuestas sicológicas llamadas KAP Studies (iniciales inglesas de knowledge, attitude y practice), tan apreciadas hace apenas 5 ó 10 años, han sido abandonadas a favor de encuestas referidas en forma casi exclusiva a los medios de contracepción y en particular el dispositivo. Quizás sea así porque se ha llegado a pensar, como se ha visto, que los deseos de limitación de los nacimientos en el Tercer Mundo están ahora firmemente establecidos y que ya es tiempo de pasar a una acción cuya urgencia no es discutida por nadie, sin preocuparse de otra cosa sino de la eficacia y aceptabilidad de los métodos contraceptivos recientemente perfeccionados.

En realidad, la necesidad de investigaciones fundamentales no ha disminuido, sino al contrario, porque siguen faltando datos indispensables para una acción bien preparada, como, por ejemplo, la influencia que pueda tener la disminución de la mortalidad infantil sobre el comportamiento concerniente a la fecundidad o sobre los cambios del medio sociocultural que puedan favorecer en una población más que en otra una disminución de la fecundidad. Presionados por el tiempo, se pasa directamente - según parece, de manera uniformea la fase de aplicación, sin obligarse antes y en cada caso a una observación encuadrada en un análisis global; se prescriben soluciones 
sin poseer siempre un conocimiento suficiente del medio en el que se interviene.

La fecundidad es un fenómeno tan complejo que no se puede uno contentar con estudiar aspectos parciales. Por eso nos parece que las investigaciones que tienden a separar los factores técnicos o sicológicos de los culturales, sin que una visión de conjunto permita separar el contenido humano de la acción, pueden decepcionar a la vez al investigador y al hombre de acción. Se debería conceder por lo menos que las encuestas tipo KAP deberían preceder a todo programa de regulación de los nacimientos, aunque no fuera sino para aumentar la eficacia. Sería sin duda deseable en muchos casos aligerar la forma de las encuestas, porque la experiencia demuestra que a menudo es necesario esperar algunos años antes de disponer de un análisis de los resultados. También los planos teóricos de estas encuestas se mejorarían al ser revisados en razón de un mejoramiento de las técnicas contraceptivas y de las modificaciones de actitudes. Es necesario un esfuerzo de renovación sobre este punto. Contentarse con discusiones en que se compara el número ideal de hijos con el número efectivo viene a ser notoriamente insuficiente. El problema no es, por supuesto, de fácil solución, pues estas encuestas recogen un conjunto de datos cuya interpretación es una de las tareas más delicadas. Pero pasar de un dato incoherente a un dato inteligible es un paso propio de todos los que trabajan en las ciencias humanas, donde lo oculto es sobre todo difícil de encontrar.

Un mejor conocimiento de los datos de población será igualmente $y$ muy pronto indispensable. La difusión de nuevos procedimientos de limitación de los nacimientos y la adopción de políticas más claras en este campo nos va a llevar a seguir paso a paso las curvas de la fecundidad. Debemos convenir en que estamos mal preparados para medir las modificaciones cuando ellas se producen, porque nos faltan estadísticas valederas. Si se puede uno contentar con el cálculo de las tasas brutas de natalidad en tanto que estas poblaciones no se alejen mucho de los modelos de población estable o cuasi estable, es evidente que esta medida se considerará insuficiente desde el momento en que las modificaciones se produzcan en la fecundidad, es decir, en el momento en que se entre en "régimen perturbado". Será, pues, cada día más urgente para los demógrafos del Tercer Mundo disponer de instrumentos de análisis aplicados a las observaciones lo mejor adaptados a su investigación. ¿ No sería conveniente que desde ahora se pusieran de acuerdo sobre los datos que permitan una buena apreciación de las perturbaciones desde que éstas aparezcan? Porque ya se conoce la dilación que exige la realización de un aparato estadístico apropiado. Las informaciones sobre los nacimientos según la edad de las madres y según el número de partos, por ejemplo, que permiten el cálculo de las probabilidades de crecimiento según el método de Henry [41], serían sin duda alguna útiles. En efecto, sería de gran interés saber en qué cohortes y a partir de qué número de hijos se modifican los comportamientos.

Pero no habría que contar con las solas estadísticas oficiales, y convendría poner al día los métodos de encuesta seguidos en los mues- 
treos de familias que permitan recoger datos sobre las crónicas de acontecimientos en las familias (casamientos, nacimientos, muertes e intervalos de tiempo entre cada acontecimiento), y en la medida de lo posible, en relación con las actitudes y los comportamientos.

\section{CONCLUSIÓN}

Presenciamos, de manera evidente, una maduración de las ideas sobre el principio mismo de la contracepción en todo el Tercer Mundo. Las resistencias de orden moral, religioso, político y, sobre todo, sicológico, que se creían difícilmente superables hace todavía pocos años, han cedido bruscamente por todas partes en el curso de los últimos años.

El progreso mismo de las técnicas anticonceptivas ha contribuido mucho a esta evolución de ideas. Existen ahora medios capaces de realizar un papel verdaderamente regulador de la fecundidad, de los cuales hemos carecido durante nuestra propia revolución demográfica en Europa. La eficacia de un procedimiento como el dispositivo intrauterino ha sido casi total, comparada con los procedimientos tradicionales, sobre todo en las mujeres que han sentido la mayor necesidad de ellos; es decir, aquellas que tienen por lo menos tres hijos.

Los motivos de esperanza, sin embargo, no están exentos de ilusión y de una cierta reticencia. Es necesario que la fisiología de la mujer y que la sociedad toleren este dispositivo que se ofrece al Tercer Mundo. (Podría decirse que la píldora está mejor adaptada al espíritu occidental, sin que se haya dado una verdadera prueba de esto.) ¿Se ha insistido demasiado y valdría más esperar para disponer de todas las informaciones suficientes, antes de lanzarse a una acción de tal importancia? Comprometerse a una actividad que se proyecta necesariamen. te a largo plazo y cuyas repercusiones pueden ser incontrolables, no es lo mismo que sentarse en un vehículo que se detiene en el lugar deseado. Una falla entre las intenciones primitivas de los actores y los resultados de la acción no sería de extrañar si las investigaciones no se han adelantado lo suficiente. A esto se ha respondido que se ha tomado conciencia demasiado tarde ya de un fenómeno que produce consecuencias cada día más difíciles de corregir.

No nos toca discutir aquí el fundamento de estas actitudes que pueden llevarnos más allá de la ciencia. Lo que nos interesa como demógrafos es la observación lo más fiel posible de este fenómeno bajo todos los aspectos, incluso los relacionados con la moral y la política, y el estudio de sus consecuencias para la colectividad.

\section{REFERENCIAS BIBLIOGRAFICAS}

[1] Jacquart, A. "La reproduction humaine en régime malthusien. Un modèle de simulation par la méthode de Monte-Carlo", Population, 22, 1967, Núm. j, pp. 897-920.

[2] Southam, A. L. "Historical Review of Intra-uterine Devices", en [9], pp. 3-5.

[3] Gräfenberg, E. "Einfluss der intrauterinen Konzeptions-Verhütung auf die Schleimhaut", Arch. Gynäk, 144, 1931, p. 345. 
-. "An Intra-uterine Contraceptive Method", en Seventh International Birth Control Conference, Zurich, septiembre de 1930. Baltimore, Williams \& Wilkins, 1931, pp. 33-47.

- "The Intra-uterine Method of Contraception", en Norman Haire (comp.), Third Congress for Sexual Reform, Londres, 8-14 de noviembre de 1929. Londres, Kegan, Trench, Trubner \& Co., 1930, apéndice, pp. 610-617.

[4] Vellay-Dalsace, A. "Les contraceptifs intra-utérins. Bibliographie: 1920-1965". [INED], 1965. Multigrafiado. (Institut National des Techniques de la Documentation, Rapport de fin d'études, Promotion 1965.)

[5] Tietze, C. "History and Statistical Evaluation of Intra-uterine Contraceptive Devices", en C. Sheps y J. C. Ridley (comp.), Public Health and Population Change; Current Research Issues. University of Pittsburg Press, 1965.

[6] Oppenheimer, W. "Prevention of Pregnancy by the Gräfenberg Ring Method: a Re-evaluation after 28 Years Experience", Amer. J. Obstet. Gynec. 78 (2), agosto de 1959 , pp. 446-454.

[7] Ishihama, A. "Clinical Studies on Intra-uterine Rings, Especially the Present State of Intra-uterine Rings', Yokohama Med. Bull., 10 (2), abril de 1959, pp. 89-105.

[8] Proceedings of the Conference on Intra-uterine Contraceptive Devices, Nueva York, 30 abril-19 mayo de 1962, Amsterdam, Excerpta Medica Foundation, 1962. (International Congress Series, Núm. 54.)

[9] Proceedings of the Second International Conference on Intra-uterine Contraception, Nueva York, 2-3 octubre de 1964. Amsterdam, Excerpta Medica Foundation, 1965. (International Congress Series, Núm. 86.)

[10] Mastroianni, L. y Hongsanand, C. "Mechanism of Action of the Intra-uterine Contraceptive Device in the Primate. I: Tubal Transport of Ova and Distribution of Spermatozoa", en [9], pp. 194-197.

[11] Mauldin, P. W., Nortman, D. y Stephan, F. F. "Retention of IUDs: An International Comparison", en Studies in Family Planning, Núm. 8, abril de 1967, pp. 1-12.

[12] Rock, J., García, C. R. y Pincus, G. "Synthetic Progestins in the Normal Human Menstrual Cycle", en Recent Progress in Hormone Research, 1957, 13, p. 323.

[13] Potter, R. G. "Additional Measures of Use-effectiveness of Contraception", Milbank Memorial Fund Quarterly, 41, octubre de 1963, pp. 400-418.

[14] Tietze, C. "Intra-uterine Contraception: Recommended Procedures for Data Analysis", en Studies in Family Planning, Núm. 18 (suplemento), abril de 1967, pp. $1-6$.

[15] Potter, R. G. "Application of Life Table Techniques to Measurement of Contraceptive Effectiveness". Ponencia presentada al Congreso Mundial de Población, Belgrado, 30 agosto-10 septiembre de 1965.

[16] Tietze, C. "Aspects statistiques de la contraception. Utilisation et efficacité", en La contraception: risques, inconvénients et facteurs d'échecs des diverses méthodes, París, Masson, 1963, pp. 231-241.

[17] Family Planning in Taïwan, Republic of China, 1965-1966. Taïchung, 1966.

[18] Davis, H. J. e Israel, R. "Uterine Cavity Measurements in Relation to Design of Intra-uterine Contraceptive Devices", en [9], pp. 135-141.

[19] Tietze, C. Cooperative Statistical Program for the Evaluation of Intra-uterine Devices. Seventh Progress Report. Septiembre de 1966. Multigrafiado.

[20] - . "Contraception with Intra-uterine Devices", Am. J. Obst. Gyn., 96, Núm. 7, diciembre de 1966, pp. 1043-1054.

[21] Cobb, J. C. y otros. "Pakistán: The Medical Social Research Project at Lulliani", Studies in Family Planning, Núm. 8, octubre de 1965, pp. 11-16.

[22] Hall, R. E. "Comparative Merits of the Spiral, Loop and Bow: a Survey of 11833 Woman-months of Experience at Sloane Hospital", en [9], pp. 6675.

[23] Tietze, C. Cooperative Statistical Program for the Evaluation of Intra-uterine Devices. Fifth Progress Report. Nueva York, febrero 25, 1965.

[24] Lee, B. M. e Isbister, J. "The Impact of Birth Control Programs on Fertility", en B. Berelson y otros (comp.), Family Planning and Population Programs. A Review of World Developments. Chicago, University of Chicago Press, 1966.

[25] Potter, R. G. y otros. "Taiwan: IUD Effectiveness in Taichung Medical Followup Study", en Studies in Family Planning, abril de 1967, Núm. 18. 
[26] Tieize, C. "Effectiveness and Acceptability of Intra-uterine Contraceptive Devices", Am. J. Public Health, 55, Núm. 12, diciembre de 1965, pp. 1874-1879.

[27] - "Acceptance of an Oral Contraceptive Program in a Large Metropolitan Area", Am. J. Obs. Gyn., 93, Núm. 1, septiembre de 1965, pp. 122-127.

[28] Southam, A. L. "Contraceptive Methods: Use, Safety and Effectiveness", en B. Berelson y otros [24], pp. 375-386.

[29] Organización Mundial de la Salud, "Aspects cliniques de l'utilisation des gestagenes oraux. Rapport d'un groupe scientifique de l'O.M.S.", Ginebra, O.M.S. Rapp. Tech., Núm. 326, 1966.

-. "Les dispositifs intra-utérins. Considérations physiologiques et cliniques. Rapport d'un groupe scientifique de l'O.M.S.", Ginebra, O.M.S. Rapp. Tech., Núm. 332, 1966.

[30] Rapport établi par la Commission créé à l'Institut national de la Santé et de la Recherche médicale pour examiner les conséquences éventuelles sur la santé de l'absorption de produits anticonceptionnels, París, 1966, multigrafiado.

[31] Asavanesa, W., Hawley, A. H. y Peng, J. Y. "Thailand", en B. Berelson y otros [24], pp. 95-104.

[32] Satterthwaite, A. P. "Experience with Oral and Intra-uterine Contraception in Rural Puerto Rico", en C. Sheps y J. C. Ridley [5], pp. 474-480.

[33] Kantner, J. F. "The Place of Conventional Methods in Family Planning Programs", en B. Berelson y otros [24], pp. 403-410.

[34] Freedman, R. "Family Planning Programs Today: Major Themes of the Conference", en B. Berelson y otros [24], pp. 811-826.

[35] Naciones Unidas, Report on the Family Planning Program in India. Nueva York, 1966, Doc. TAD/IND/48.

[36] Tabah, L. y Samuel. "Preliminary Findings of a Survey of Fertility and Attitudes Towards Family Formation in Santiago de Chile", en Research in Family Planning, Princeton, Princeton University Press, 1962.

[37] Berelson, B. "KAP Studies on Fertility", en B. Berelson y otros [24], pp. 655-668.

[38] Tabah, L. "Plan de recherche de sept enquêtes comparatives sur la fécondité en Amérique latine", Population, 19, 1964, Núm. 1, pp. 95-126.

[39] Miró, C. "Some Misconceptions Disproved: A Program of Comparative Fertility Surveys in Latin America", en B. Berelson y otros [24], pp. 615-632.

[40] Organización Mundial de la Salud, "Immunologie et reproduction humaine. Rapport d'un groupe scientifique de l'O.M.S.", Ginebra, O.M.S. Rapp. Tech., 1966, Núm. 334.

[41] Henry, L. "Fécondité des mariages. Nouvelles méthodes de mesure". Paris, INED, Travaux et Documents, Cuaderno Núm. 16, 1953. 